\title{
Numerical Experiments in Fourier Asymptotics of Cantor Measures and Wavelets
}

\author{
Prem Janardhan, David Rosenblum and Robert S. Strichartz
}

\section{CONTENTS}

\section{Introduction}

2. One-term Recursion for Integer $\rho$

3. One-term Recursion for Noninteger $\rho$

4. Two-term Recursion: The Commensurable Case

5. Two-term Recursion: The Incommensurable Case

6. Julia Sets

7. Experimental Procedure

References
Janardhan and Rosenblum were supported by the National Science Foundation's Research Experiences for Undergraduates Program. Strichartz was supported in part by the National Science Foundation grant DMS-9103348.
We discuss the asymptotic behavior of Fourier transforms of Cantor measures and wavelets, and related functions that might be called multiperiodic because they satisfy a simple recursion relation involving a blend of additive and multiplicative structures.

Our numerical experiments motivated conjectures about this asymptotic behavior, some of which we can prove. We describe the experiments, the proofs, and several remaining conjectures and open problems. We also contribute to the evolving iconography of fractal mathematics by presenting the numerical evidence in graphical form.

\section{INTRODUCTION}

An interesting class of functions, which might be called multiperiodic, can be described by simple recursion relations involving a blend of additive and multiplicative structures. Such functions arise typically as Fourier transforms of self-similar objects, such as Cantor measures and wavelets. We report here the results of numerical experiments we performed on these functions, and some theorems and conjectures about their asymptotic behavior that were directly inspired by the numerical evidence.

We study functions of one variable, which we denote $F(x)$ and normalize to satisfy $F(0)=1$. The simplest case we consider is a one-term recursion

$$
F(x)=f\left(\frac{x}{\rho}\right) F\left(\frac{x}{\rho}\right)
$$

where the scaling factor $\rho$ satisfies $\rho>1$ and $f$ is a periodic function of period one satisfying the consistency condition

$$
f(0)=1
$$


This leads easily to the infinite-product representation

$$
F(x)=\prod_{k=1}^{\infty} f\left(\frac{x}{\rho^{k}}\right),
$$

which converges rapidly as long as $f$ is $C^{1}$ near zero. We devote Sections 2 and 3 to this class of functions, for $\rho$ an integer and not an integer, respectively.

In Sections 4 and 5 we examine solutions of twoterm recursion relations

$$
F(x)=f_{1}\left(\frac{x}{\rho_{1}}\right) F\left(\frac{x}{\rho_{1}}\right)+f_{2}\left(\frac{x}{\rho_{2}}\right) F\left(\frac{x}{\rho_{2}}\right),
$$

where $\rho_{1}, \rho_{2}>1$ and the consistency condition is now

$$
f_{1}(0)+f_{2}(0)=1 .
$$

There is no analog of the product expansion (1.2), but it is easy to see that there is a unique continuous solution to (1.3) with $F(0)=1$ if $f_{1}$ and $f_{2}$ are $C^{1}$.

In Section 6 we examine the Fourier transforms of the equilibrium measure on the Julia sets for the mapping $z \mapsto z^{2}-r$, where $r>2$, and look for analogous asymptotic behavior, although these functions are no longer multiperiodic.

The simplest example of a product of the form $(1.2)$ is

$$
\sum_{k=1}^{\infty} \cos \frac{\pi x}{2^{k}}=\frac{\sin \pi x}{\pi x},
$$

an equality due to Euler (although special cases were known to Viète almost 400 years ago). The right side of (1.5) is recognized as the Fourier transform of Lebesgue measure on the interval $\left[-\frac{1}{2}, \frac{1}{2}\right]$,

$$
\int_{-1 / 2}^{1 / 2} e^{2 \pi i x y} d y=\frac{\sin \pi x}{\pi x},
$$

while the left side expresses the fact that this measure is obtainable by repeatedly halving the interval and assigning equal probability to each half. Another way of saying this is that the recursion relation (1.1) for this example is equivalent to the self-similarity of the measure

$$
\mu(E)=\frac{1}{2} \mu\left(\frac{1}{2} E-\frac{1}{4}\right)+\frac{1}{2} \mu\left(\frac{1}{2} E+\frac{1}{4}\right) .
$$

Of course, the function (1.5) exhibits an especially simple asymptotic behavior - see Figure 1-and it would be misleading to expect the same from the other functions we study. In Section 2 we will be able to "explain" this behavior in terms of certain remarkable "coincidences" involving the integral

$$
\int_{0}^{1} \log \cos ^{2} \pi x d x
$$

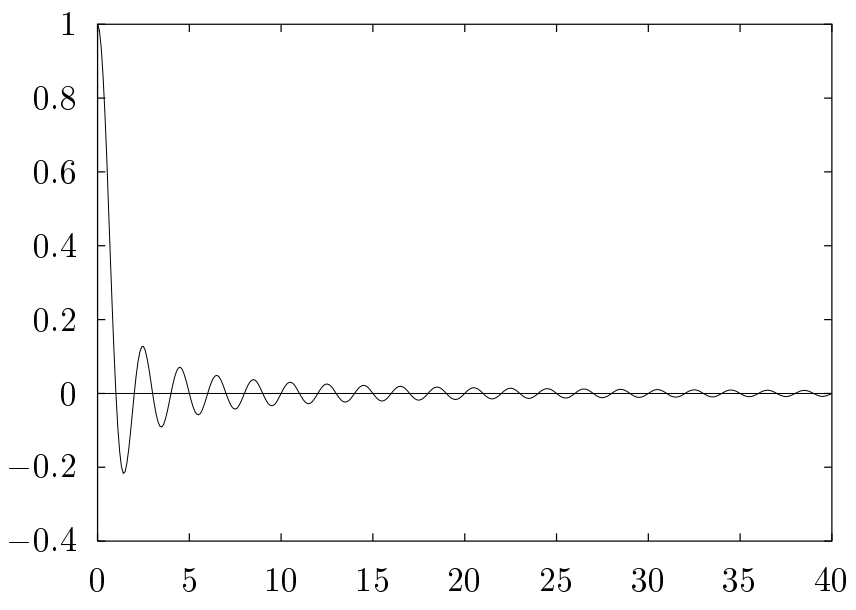

FIGURE 1. The simplest example of a function satisfying $(1.1)$ is $F(x)=(\sin \pi x) /(\pi x)$, obtained by setting $f(x)=\cos \pi x$ and $\rho=2$.

If we keep the same function $f(x)=\cos \pi x$ but take $\rho>2$ in (1.2), we get the Fourier transform of the Cantor measure on $\left[-\frac{1}{2}, \frac{1}{2}\right]$ obtained by deleting the middle segment of length $1-2 / \rho$, assigning equal probability to the outer segments and iterating. The value $\rho=3$ corresponds to the usual middle-third Cantor set; Figure 2 shows the graph of $F(x)$ in this case.

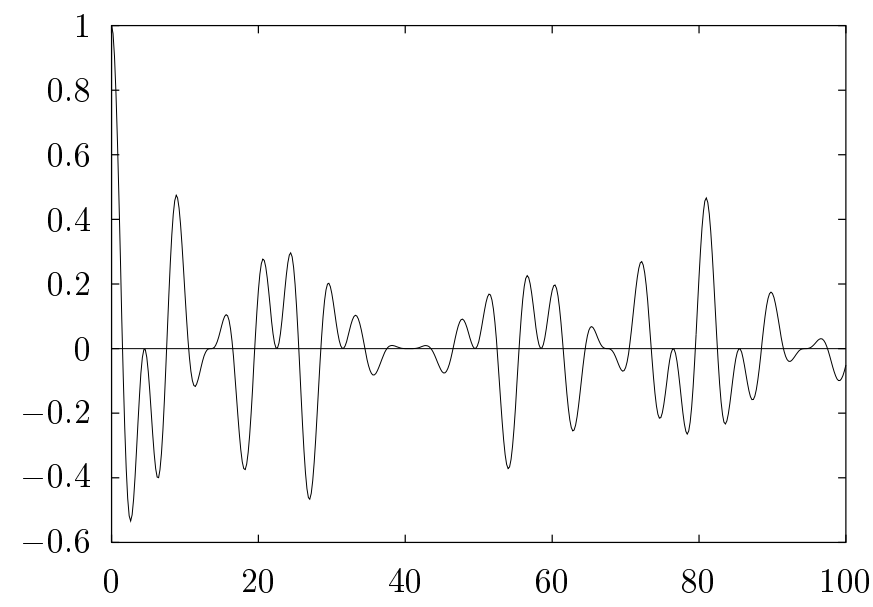

FIGURE 2. Solution of (1.1) with $f(x)=\cos \pi x$ and $\rho=3$. 

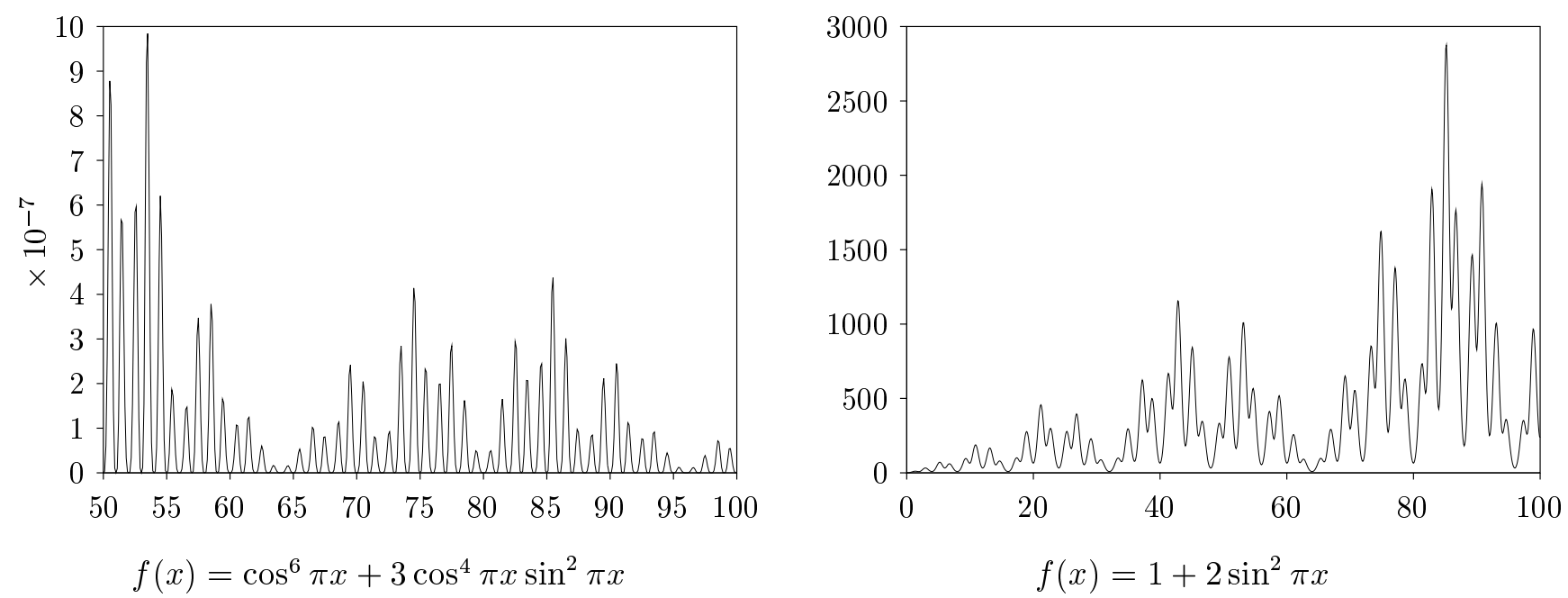

FIGURE 3. Graphs of $F(x)$ for $\rho=2$ and the indicated functions $f(x)$.

These functions have been extensively studied since the thirties [Wiener and Wintner 1939; Erdös 1940; Salem 1983; Strichartz 1990; Strichartz 1993; Lau; Lau and Wang]. More generally, the Fourier transforms of any of the self-similar measures defined in [Hutchinson 1981] will satisfy multiterm recursion relations (1.3) with the $f_{i}(x)$ trigonometric polynomials. (In general, there may be more than two terms, but we expect that the two-term case already exhibits all the behaviors of the general case.)

Another source of functions of the form (1.2) is the theory of wavelets. If $\varphi(x)$ is the scaling function for the compactly supported wavelets of Daubechies [1988, 1992], then $F(x)=|\hat{\varphi}(x)|^{2}$ satisfies (1.2) with $\rho=2$ and $f(x)$ a nonnegative trigonometric polynomial such that

$$
f(x)+f\left(x+\frac{1}{2}\right) \equiv 1 .
$$

It is easy to construct such $f$ : simply take the first half of the terms in the binomial expansion of $\left(\cos ^{2} \pi x+\sin ^{2} \pi x\right)^{2 N+1}$. For the theory of wavelets, it is important to obtain estimates of the form

$$
|F(x)| \leq c|x|^{-\alpha}
$$

since such estimates imply smoothness of the wavelets of order $\alpha-1-\varepsilon$. See [Daubechies 1992, Chap. 7] for a detailed discussion and references.

The goal of our experiments was to elucidate the asymptotic behavior of the function $F(x)$ as $x \rightarrow$ $\infty$. From previous work, we knew that we could expect relatively simple behavior on the part of integrals such as

$$
I_{q} F(x)=\int_{0}^{x}|F(t)|^{q} d t .
$$

For example, in [Strichartz 1990; Strichartz 1993; Lau; Lau and Wang], it is proved under various hypotheses that, as $x \rightarrow \infty$, the function $I_{2} F$ behaves like $c x^{-\alpha}$ or $\rho(x) x^{-\alpha}$, for a specific value of $\alpha$, where $\rho(x)$ is a multiplicative periodic function. Furthermore, numerical experiments performed by Maria Korolov showed that we can expect the same for $I_{q} F$ for any $q>0$ (we report some related experiments in Section 5).

The problem we considered initially was this:

Problem 1.1. Find some conditions on the pointwise asymptotic behavior of $F$ that would imply the known and conjectured asymptotic behavior of $I_{q} F$.

We did not solve this problem, but we did discover some fascinating pointwise asymptotic behavior for $F$. The results for the one-term recursion relation (1.2) are completely proven in this paper, but for the general case we can only present a conjecture.

Our first approach was more exploratory than experimental, in that Problem 1.1 does not present any conjecture that can be tested. For simplicity, we restricted attention to functions $f$ that are nonnegative (replacing $f$ by $|f|$ just replaces $F$ by $|F|$, so we obtain the asymptotic behavior of $|F|$ in the general case). We also used trigonometric polynomials for $f$, so the resulting $F$ is band-limited $(\hat{F}$ 
has compact support) and therefore is slowly oscillating, which makes it easier to graph accurately.

We plotted the graphs of a number of functions $F$, some of which are shown in Figures 2-3 and Figure 11 in Section 3. After some trial-and-error manipulations of these graphs, we were led to consider the sequence of functions

$$
\frac{1}{n} \log F\left(\rho^{n} x\right)
$$

on the interval $1 \leq x \leq \rho$. Figure 4 shows the graphs of these functions for $F$ and $\rho$ as in Figure 3. (Actually these figures plot a slight variant of (1.6): see (2.2).) Figures 5-6 in Section 2 and 12-13 in Section 3 provide other examples.

It is easy to conjecture from this evidence that the sequence (1.6) converges as $n \rightarrow \infty$ in some weak sense to something quite complicated. But actually, we will prove under minimal hypotheses that the sequence always converges almost everywhere and in $L^{p}$ norm to the constant $\int_{0}^{1} \log f(t) d t$. The convergence does not take place everywhere; in fact, there is a dense set of points where the sequence converges to a value different from this constant, which explains why the graphs appear so complicated.

One striking feature of the graphs of (1.6) when $\rho$ is an integer, which is not present otherwise, is a strong appearance of self-similarity. To explain what is going on, we need a new definition.

Definition 1.2. A sequence of functions $h_{n}(x)$ on a domain $D$ is said to be asymptotically self-similar with respect to a transformation $T: D_{0} \rightarrow D$, where $D_{0}$ is a subset of $D$, if for every $\varepsilon>0$ there exists $N$ such that

$$
\left|h_{n}(T x)-h_{n}(x)\right| \leq \varepsilon
$$

for all $n \geq N$ and all $x \in D_{0}$.

Note. This definition probably should be generalized to allow a transformation of the range as well as the domain, but for the sake of simplicity, we have restricted ourselves to the above, which is adequate for our examples.

One of our main results is that the sequence (1.6) with $\rho$ an integer is asymptotically self-similar with respect to the transformation

$$
T x=\rho x(\bmod 1) .
$$

To see this, we need to look at the graphs of $h_{n}(x)$ and $h_{n}(T x)$ and observe that they differ by a small amount for large $n$. However, we come up against a serious paradox - we might almost say an uncertainty principle - when we do this. To make $\varepsilon$ small, we have to take $n$ large. But the larger we take $n$, the more complicated the graph of $h_{n}$ becomes, and the harder it is to see anything at all. In fact, then, we settle for a compromise, where $n$ is only moderately large, so the complicated oscillations of $h_{n}$ are still visible, and $\varepsilon$ is only moderately small, so the difference between $h_{n}(x)$ and $h_{n}(T x)$ is still noticeable, but not too large.

It follows immediately from the definition that if a sequence $h_{n}(x)$ is asymptotically self-similar with respect to $T$, it is also asymptotically self-similar with respect to every power $T^{k}$ of $T$ (on a suitable subdomain). Thus our functions $h_{n}$ will exhibit approximate self-similarity with respect to a whole family of transformations. However, with $n$ fixed, the error $\varepsilon$ in (1.7) will grow with $k$, so the difference between the graphs of $h_{n}(x)$ and $h_{n}\left(T^{k} x\right)$ will become more and more noticeable (see Figure 7 for an illustration). Nevertheless, this multiple selfsimilarity is very striking (see Figure 4).

The case of the one-term recursion relation with noninteger $\rho$, discussed in Section 3, reveals the same convergence properties for the sequence (1.6), but the asymptotic self-similarity is lost. For the two-term recursion relation (1.3), a dichotomy was predicted by previous work [Strichartz 1993; Lau]. If $\rho_{1}$ and $\rho_{2}$ are exponentially commensurable, that is, if $\rho_{1}=\rho^{j}$ and $\rho_{1}=\rho^{k}$ for some $\rho>1$ and integers $j, k$, the two-term recursion relation leads to functions $F$ that resemble solutions to the oneterm recursion relation with scaling function $\rho$. In Section 4 we discuss the experimental evidence for this.

Without the assumption of exponential commensurability, we have not been able to discern any pointwise asymptotic regularity for $F$. In Section 5 we report on experimental evidence for the asymptotic behavior of the integrals $I_{q} F$ of (1.6). The question can be formulated as follows.

Problem 1.3. Under what circumstances is it true that

$$
I_{q} F(x) \sim c_{q} x^{b_{q}}
$$

as $x \rightarrow \infty$ ? 

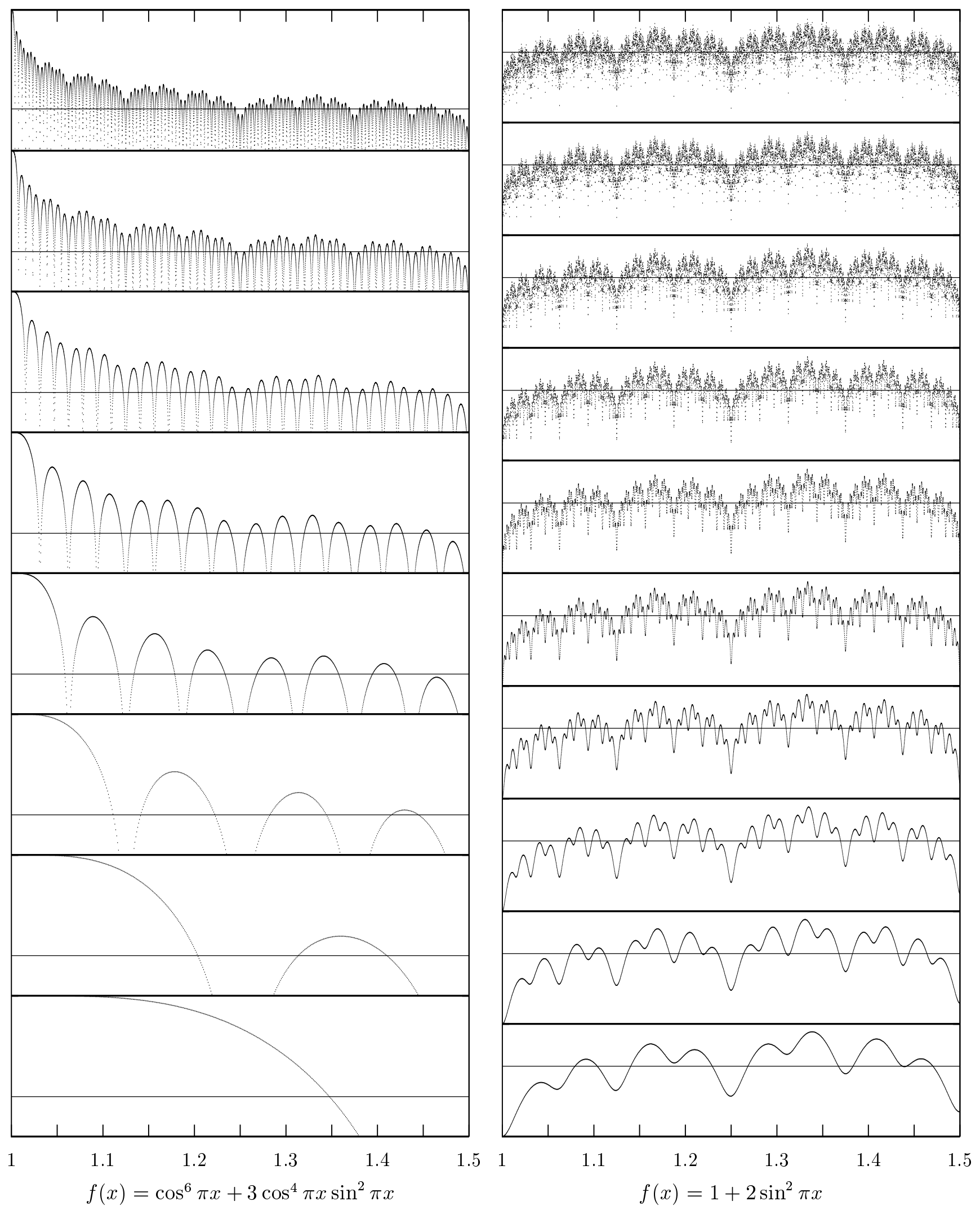

FIGURE 4. Graphs of $h_{n}(x)=n^{-1}\left(\log F\left(\rho^{n} x\right)-\log F(x)\right)$ for the functions $F$ of Figure 3 and $\rho=2$, for $n$ increasing (as we go up) from 1 to 8 on the left and from 5 to 14 on the right. (On the left, only values $h_{n}(x) \geq-3$ are shown.) The graphs are symmetric with respect to the lines $x=1$ and $x=1.5$ (in particular, $h_{n}$ is periodic of period 1). The horizontal lines indicate the $\operatorname{limit}_{n \rightarrow \infty} h_{n}(x)$, valid almost everywhere. 
The evidence we obtain does not allow us to conjecture an answer, but we do produce a conjecture that the weaker statement

$$
\lim _{x \rightarrow \infty} \frac{\log I_{q} F(x)}{\log x} \quad \text { exists }
$$

holds in great generality.

In Section 6 we report on numerical evidence for the asymptotic behavior (1.8) or (1.9) when $F$ is the Fourier transform of the equilibrium measure on a Julia set. The value of $b_{2}$, if it exists, is related to the mean quadratic variation of the measure, according to results in [Lau and Wang]. We have been able to compute both values approximately, and they agree to within the error of our method. In [Strichartz 1992b], it is proved that the limit (1.9) exists for $q=2$.

In Section 7 we discuss the programs that were used to generate the data, some of the problems that arose in creating the programs, and the tests of accuracy that we performed.

We are grateful to Rick Durrett for discussions concerning probability theory, to John Hubbard for advice on generating the Julia-set measures, and to Peter Sarnak for suggesting the use of the ergodic theorem in Corollary 2.2.

\section{ONE-TERM RECURSION FOR INTEGER $\rho$}

In this section,

$$
F(x)=\prod_{k=1}^{\infty} f\left(\frac{x}{\rho^{k}}\right)
$$

for $\rho$ an integer. We specify $F$ by giving $\rho$ and $f$.

We have already seen some important examples. In Figure 2 we had $\rho=3$ and $f(x)=\cos \pi x$, and $F$ was the Fourier transform of the usual Cantor measure on the interval $\left[-\frac{1}{2}, \frac{1}{2}\right]$.

In Figure 3 (left) we had $\rho=2$ and

$$
f(x)=\cos ^{6} \pi x+3 \cos ^{4} \pi x \sin ^{2} \pi x .
$$

Then $F=|\hat{\varphi}|^{2}$, where $\varphi$ is the scaling function for a compactly supported wavelet. We restricted the graph to $50 \leq x \leq 100$, because the function falls off so rapidly that it is impossible to see much detail if we take an interval starting at $x=0$. This is the problem with the graphs presented in [Daubechies 1988].

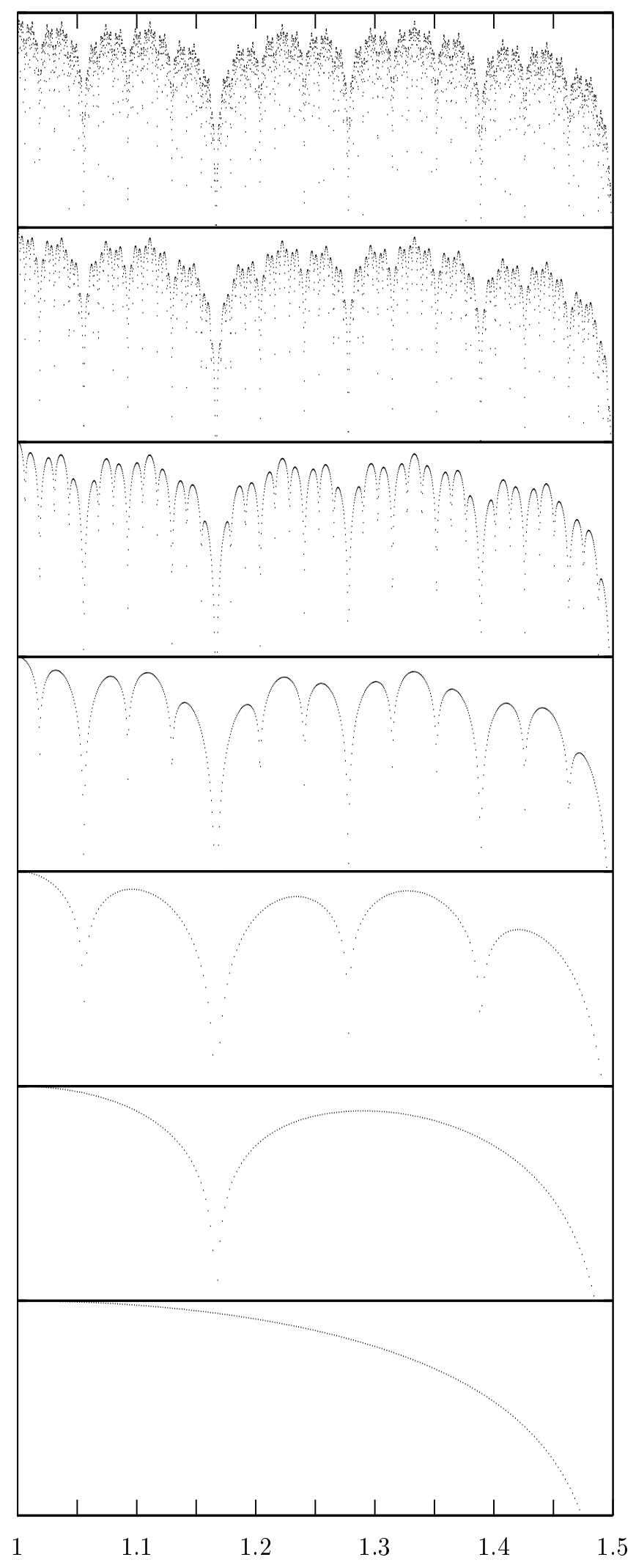

FIGURE 5. Graphs of $h_{n}(x)$, for $1 \leq n \leq 7$, for the function $F$ arising from $f(x)=\cos ^{2} \pi x$ and $\rho=3$ (the square of the function in Figure 2). Large negative values of $h_{n}(x)$ have been excised. 


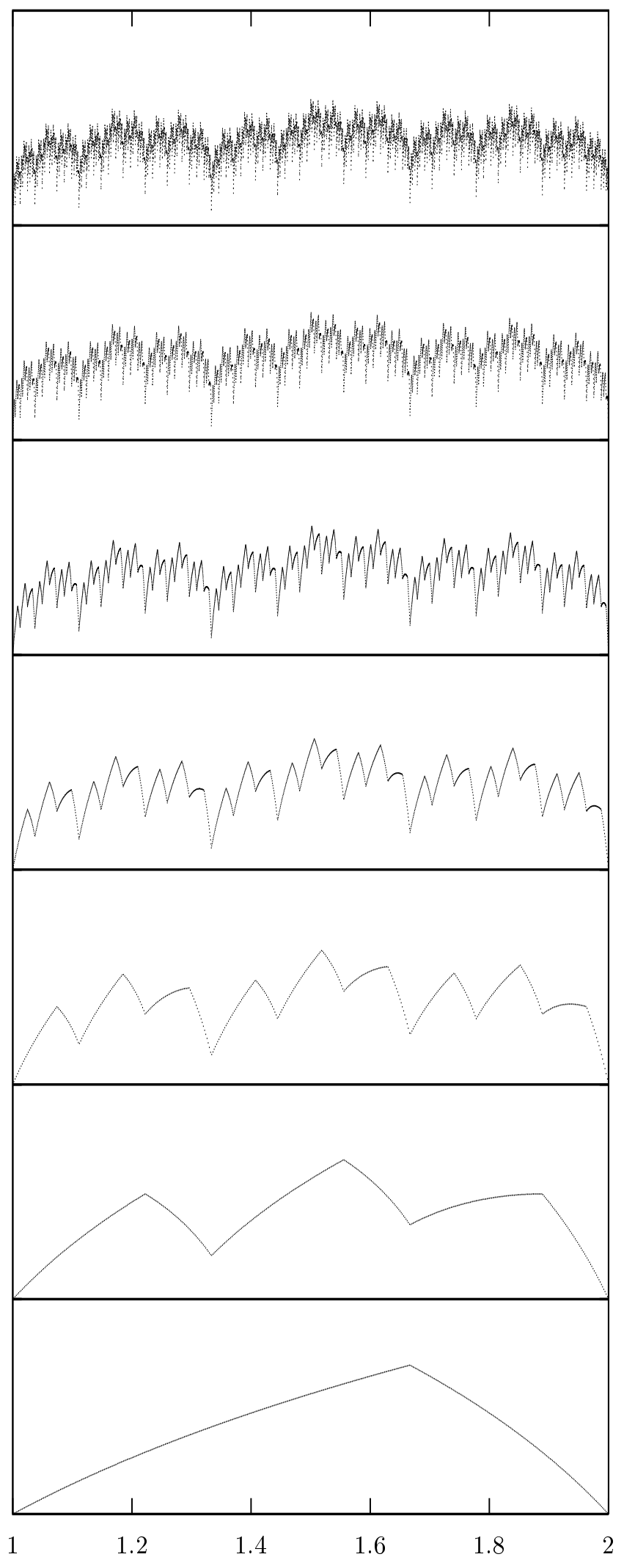

FIGURE 6. Graphs of $h_{n}(x)$, for $1 \leq n \leq 7$, for the function $F$ arising from an asymmetric sawtooth function $f$ and $\rho=3$.
Note that this $f(x)$ has a factor $\cos ^{4} \pi x$, and we know from Euler's formula (1.5) that this contributes a factor of $(\sin \pi x / \pi x)^{4}$ to $F(x)$. If we remove the factor $\cos ^{4} \pi x$, we are left with $f(x)=$ $1+2 \sin ^{2} \pi x$, which leads to Figure 3 (right), still with $\rho=2$. The function $F$ now grows with $x$ rather than decays. Because $f(x)$ is bounded away from zero in this example, the behavior of $F(x)$ is a bit simpler, and we can already discern in the figure a hint of the asymptotic behavior we are looking for.

It is tempting to take the logarithm of (2.1) to turn the product into a sum. In order to do this we must assume that $f(x)$ is nonnegative; if this is not the case to begin with, as in Figure 2, we can always take the square $f(x)^{2}$ in place of $f(x)$, which simply squares $F(x)$. We observe then that

$$
\log F\left(\rho^{n} x\right)-\log F(x)=\sum_{j=0}^{n-1} \log f\left(\rho^{j} x\right)
$$

and therefore we define

$$
h_{n}(x)=\frac{1}{n} \sum_{j=0}^{n-1} \log f\left(\rho^{j} x\right)
$$

Since $h_{n}(x)$ differs from $\frac{1}{n} \log F\left(\rho^{n} x\right)$ by $\frac{1}{n} \log F(x)$, the two functions have the same behavior as $n \rightarrow$ $\infty$ if we restrict $x$ to a fixed interval, which we take to be $1 \leq x \leq \rho$. The advantages of using $h_{n}(x)$ rather than $\frac{1}{n} \log F\left(\rho^{n} x\right)$ are that $h_{n}(x)$ is periodic of period one (here we use the fact that $\rho$ is an integer) and it is easier to compute.

In Figure 4 we show the graphs of $h_{n}$ for the wavelet scaling function of Figure 3-both the original and the factored versions. (The domain is reduced to $1 \leq x \leq 2$ because the functions are periodic of period one.) In Figure 5 we show $h_{n}$ for the Fourier transform of the Cantor measure squared, the square of Figure 2. Finally, in Figure 6, we show $h_{n}$ for an asymmetric sawtooth function $f$ and $\rho=3$. This function has no particular significance, but it is displayed to show that neither symmetry nor smoothness of $f$ play an important role in the qualitative behavior of the sequence $h_{n}$.

Despite the colorful behavior of the graphs of $h_{n}$, we have the following banal result. 
Proposition 2.1. Suppose $\rho$ is an integer and $f$ is a continuous periodic function satisfying $f>0$. Then

$$
\lim _{n \rightarrow \infty} h_{n}(x)=\int_{0}^{1} \log f(t) d t
$$

on $[1, \rho]$, almost everywhere and in $L_{p}$ norm for any $p$ with $1 \leq p<\infty$.

Proof. By the central limit theorem, the sequence of points $\rho^{j} x$ is uniformly distributed mod 1 for almost every $x$. For each such $x,(2.3)$ is merely the statement that the Monte Carlo approximations to the integral of $\log f(x)$ converge, which by a theorem of Weyl only requires Riemann integrability. $L_{p}$-convergence follows from the dominated convergence theorem, since the sequence $h_{n}$ is uniformly bounded.

Corollary 2.2. Suppose $\rho$ is an integer, $f \geq 0$ and $\log f(x) \in L^{p}$ on $[1, \rho]$, for some $p$ with $1 \leq p<\infty$. Then (2.3) holds in $L^{p}$ norm and almost everywhere.

Proof. The transformation $x \mapsto \rho x(\bmod 1)$ on $[1, \rho]$ is measure-preserving and ergodic, so the result follows by the ergodic theorem.

To understand the graphs we have obtained, we have to realize that the pointwise limit is not the constant function $\int_{0}^{1} \log f$, but rather an everywhere discontinuous function that is equal to this constant almost everywhere, and is undefined on a set of measure zero that is also dense. Suppose, to be specific, that $\rho=2$ and $x=p / q$ is a rational number with $q$ odd. Then the sequence $2^{j} p / q$ (mod 1) cycles periodically through a finite set of rationals $a_{1} / q, a_{2} / q, \ldots, a_{m} / q$, and

$$
\lim _{n \rightarrow \infty} h_{n}(x)=\frac{1}{m} \sum_{j=1}^{m} \log f\left(\frac{a_{j}}{q}\right) .
$$

(If $q$ is even, we have a similar result.) In fact, the rate of convergence in (2.4) is $O\left(n^{-1}\right)$, whereas the rate of convergence in (2.3) is roughly $O\left(n^{-1 / 2}\right)$. There is no reason why the sum in (2.4) should equal the integral $\int_{0}^{1} \log f$ exactly, and this accounts for the wild oscillations of $h_{n}$. If we try to take $n$ large enough that $h_{n}(x)$ is close to the limit, the oscillations will be so violent that the graph will be unintelligible.
The approximate self-similarity of the graphs of $h_{n}$ is striking. Perhaps Figure 6 is the best example to look at, because all the other graphs have additional symmetries due to the fact that $f(x)$ is an even function about $x=\frac{1}{2}$. Let $I_{k}$ denote the interval

$$
1+\frac{(\rho-1) k}{\rho} \leq x \leq 1+\frac{(\rho-1)(k+1)}{\rho}
$$

for $k=0, \ldots, \rho-1$ and let the transformation $T_{k}$ be defined by $x \mapsto \rho x-(k+1)(\rho-1)$. Note that $I_{k} \subset[1, \rho]$ and $T_{k}$ maps $I_{k}$ one-to-one onto $[1, \rho]$.

Proposition 2.3. Under the hypotheses of Proposition 2.1, the sequence $h_{n}(x)$ is asymptotically selfsimilar with respect to the mapping $T_{k}$ on $I_{k}$. Under the weaker hypothesis of Corollary 2.2, we have an $L^{p}$-variant of the conclusion, namely

$$
\left(\int_{I_{k}}\left|h_{n}(x)-h_{n}\left(T_{k} x\right)\right|^{p} d x\right)^{1 / p}=O\left(n^{-1}\right) .
$$

Proof. Because $f$ is periodic, we have

$$
\log f\left(\rho^{j} T_{k} x\right)=\log f\left(\rho^{j+1} x\right),
$$

so

$$
h_{n}\left(T_{k} x\right)=\frac{1}{n} \sum_{j=1}^{n} \log f\left(\rho^{j} x\right),
$$

hence

$$
h_{n}(x)-h_{n}\left(T_{k} x\right)=\frac{1}{n}\left(\log f(x)-\log f\left(2^{n} x\right)\right) .
$$

The result follows by taking the sup norm or the $L^{p}$ norm.

We illustrate the proposition in Figure 7. For the factored wavelet function $f=1+2 \sin ^{2} x$ of Figure 3 (right), we graph $h_{12}(x)$ and its composition with $T_{1}, T_{1}^{2}$ and $T_{1}^{3}$.

Figure 8 gives an idea of how slow the convergence is. For each indicated $n$, we sampled the values of $h_{n}(x)$ at $2048=2^{11}$ equally spaced points in the interval $1 \leq x \leq 2$. We sorted the resulting values and plotted them in decreasing order. Thus the plots give the cumulative distribution of values of $h_{n}$, the abcissas corresponding to the accumulated frequency and the ordinates to the values.

If $h_{n}$ were close to the limit, the curve would run close to the horizonal line at height

$$
\int_{0}^{1} \log \left(1+2 \sin ^{2} \pi t\right) d t \approx .6238107,
$$




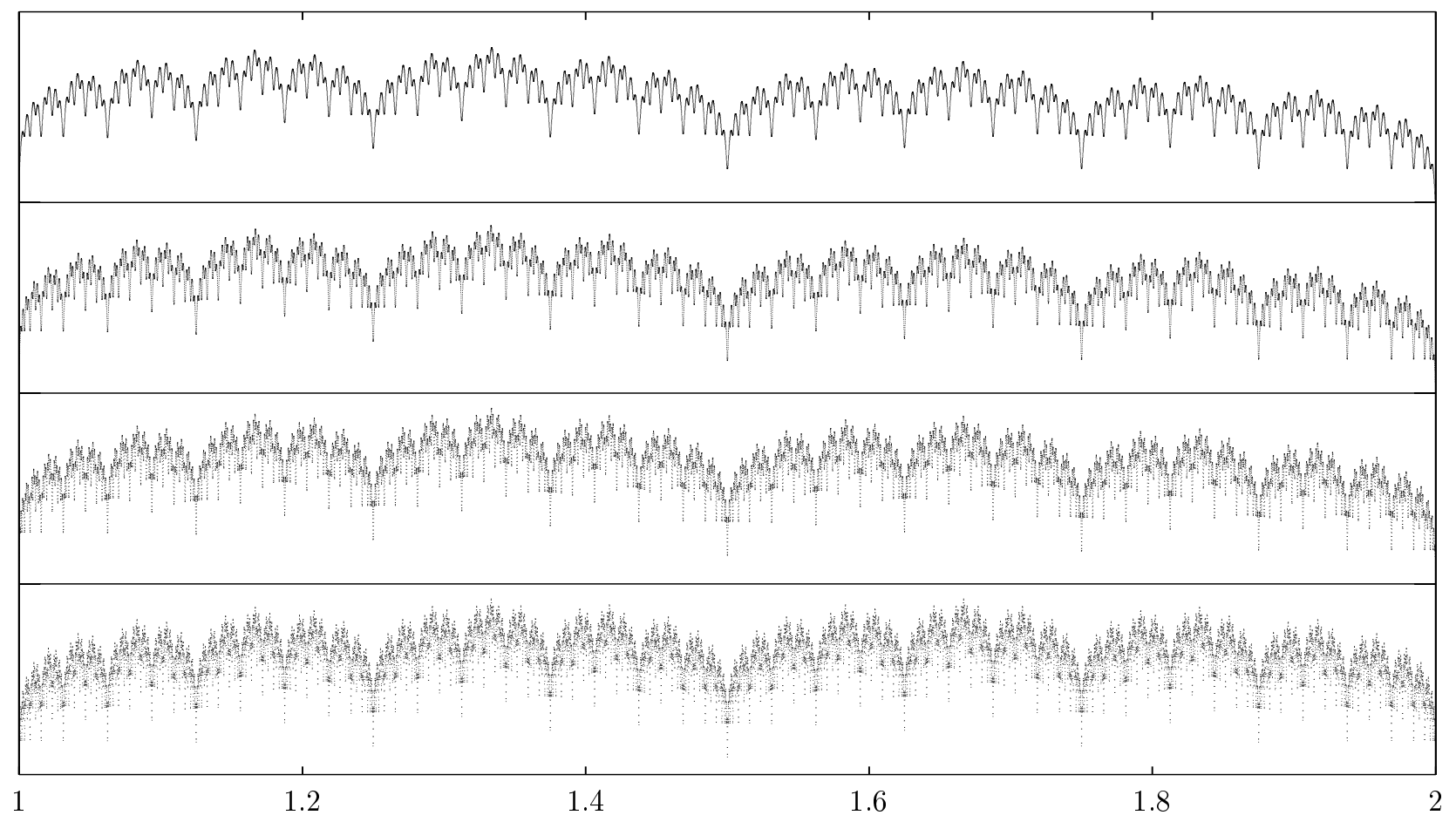

FIGURE 7. For the function $F$ arising from $f(x)=1+2 \sin ^{2} \pi x$ and $\rho=2$, the graphs of $h_{12}(x), h_{12}\left(T_{1} x\right)$, $h_{12}\left(T_{1}^{2} x\right)$ and $h_{12}\left(T_{1}^{3} x\right)$, as we go up. To get each graph from the one below it, zoom in on the right half.

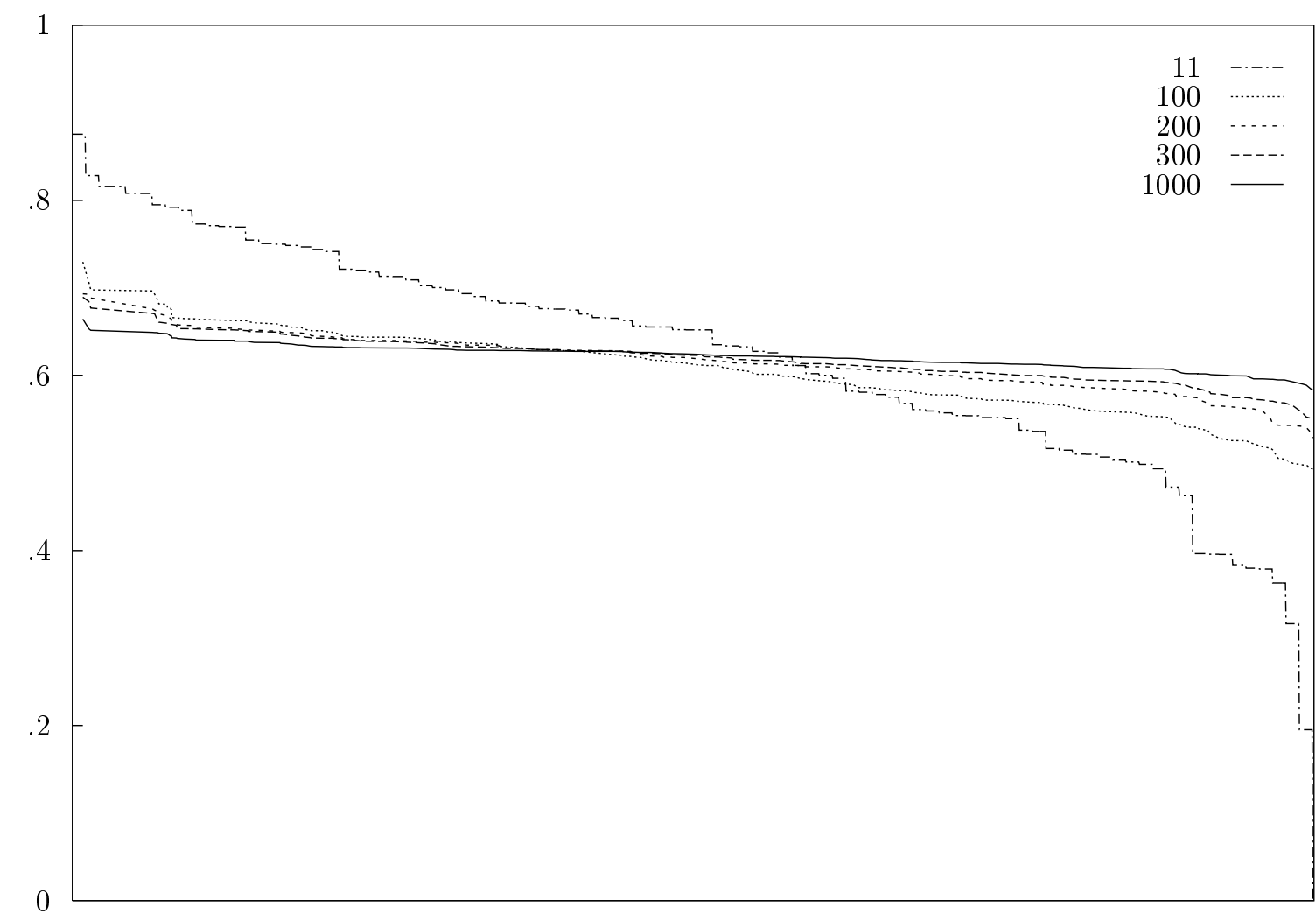

FIGURE 8. Cumulative distribution of values of $h_{n}(x)$ for the function $F$ of Figure 3 (right) in the interval $1 \leq x \leq 2$. The functions were sampled at $2048=2^{11}$ regularly spaced points. The distributions change very slowly as $n$ increases: for example, the graphs for $n=11,12,13,14$ largely overlap. 
except for a small region of larger values near the left end and a small region of smaller values near the right end. The fact that we don't see this indicates that we are quite far from convergence. It is only for $n=1000$ that we are beginning to see the expected behavior. (However, the data for high $n$ cannot be highly accurate, not only because of the difficulty in computing $h_{n}$, but because the number of sampled points is much too small.)

Figure 9 shows the graph of $h_{8}$ for $\rho=2$ and $f(x)=\cos ^{2} \pi x$, which by Euler's formula yields $F(x)=(\sin \pi x / \pi x)^{2}$. The line patterns that appear in the graph are artifacts of the regular scan used to produce it; see Section 7. The form of $F$ indicates that the regularity of $h_{n}$ is to be expected, but we can also give an explanation that is in keeping with (2.3) and (2.4).

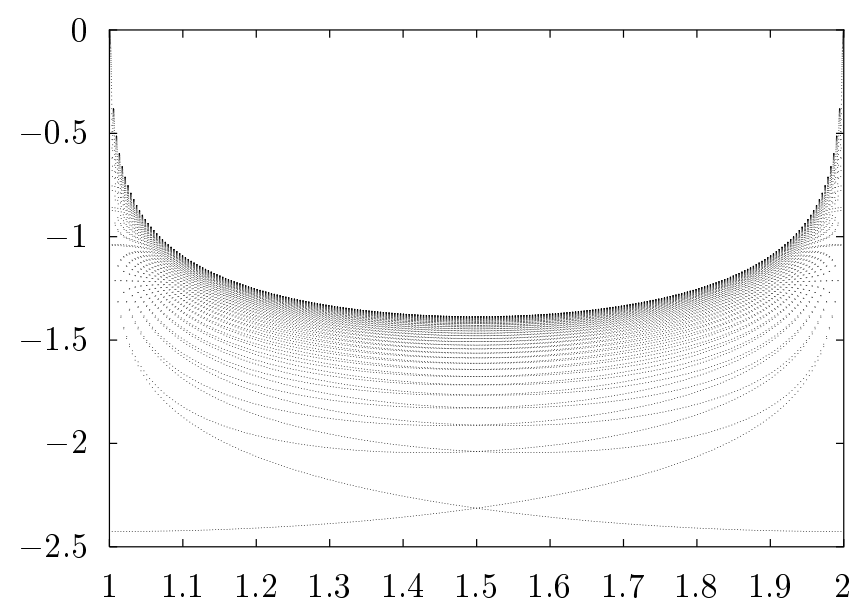

FIGURE 9. Graph of $h_{8}(x)$ for the choice $\rho=2$ and $f(x)=\cos \pi x$, obtained by sampling at $2^{14}$ regularly spaced points. Values below -2.5 have been excised.

Proposition 2.4. Let $a_{1} / q, \ldots, a_{m} / q$ be the full cycle of $2^{j} p / q(\bmod 1)$, where $p$ and $q$ are odd. Then

$$
\frac{1}{m} \sum_{j=1}^{m} \log \cos ^{2} \frac{\pi a_{j}}{q}=\int_{0}^{1} \log \cos ^{2} \pi t d t=-2 \log 2 .
$$

Proof. The value of the integral is elementary. The assertion about the sum is equivalent to

$$
\prod_{j=0}^{m-1} \cos ^{2} \frac{\pi 2^{j} p}{q}=2^{-2 m}
$$

or to

$$
\prod_{j=0}^{m-1}\left(\exp \frac{i \pi 2^{j} p}{q}+\exp \frac{-i \pi 2^{j} p}{q}\right)^{2}=1,
$$

so it suffices to show that

$$
\prod_{j=0}^{m-1}\left(\exp \frac{2 \pi i 2^{j} p}{q}+1\right)=1 .
$$

Expanding this product, we obtain simply

$$
\sum_{k=0}^{2^{m}-1} e^{2 \pi i k p / q}
$$

But $e^{2 \pi i p / q}=\omega$ is a $q$-th root of unity, and $m$ is chosen so that $2^{m} p=p(\bmod q)$, so this sum consists of $\left(2^{m}-1\right) p / q$ repetitions of $1+\omega+\cdots+$ $\omega^{q-1}=0$ followed by $\exp \left(2 \pi i\left(2^{m}-1\right) p / q\right)=1$.

More generally, any rational number can be written in the form $2^{-k} p / q$, where $p$ and $q$ are odd and relatively prime. If $k \geq 1$ and $q=1$, we obtain $h_{n}\left(2^{-k} p\right)=-\infty$ for $n$ large enough. Otherwise, it follows from (2.4) and (2.5) that

$$
h_{n}\left(2^{-k} p / q\right)=-2 \log 2+O\left(n^{-1}\right) .
$$

Figure 10 shows the graph of $h_{6}(x)$ for the choice $\rho=2$ and $f(x)$ equal to the square wave function

$$
f(x)= \begin{cases}1 & \text { if } 0 \leq x<\frac{1}{2} \\ e & \text { if } \frac{1}{2} \leq x \leq 1\end{cases}
$$

so that $h_{n}(x)$ is the average of the first $n$ digits in the binary expansion of $x$.

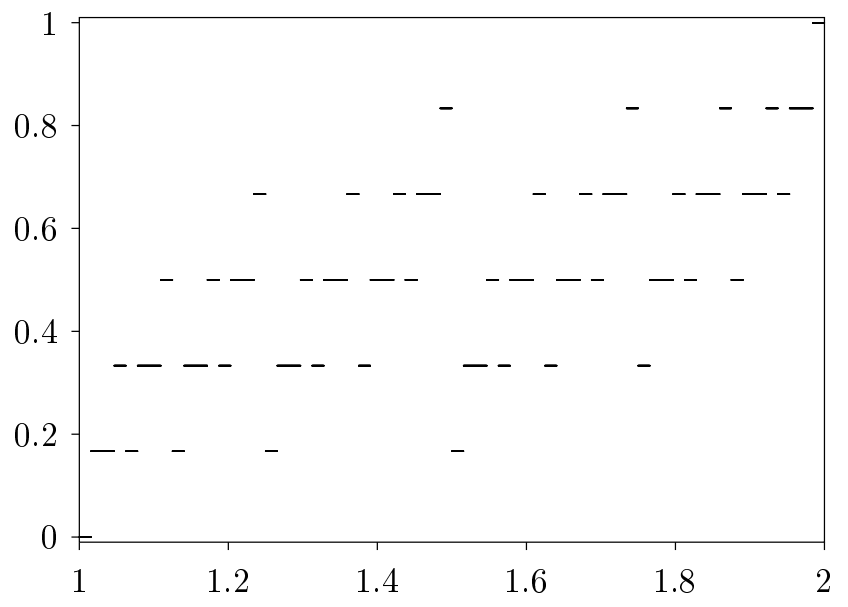

FIGURE 10. Graph of the average of the first six binary digits in the binary expansion of the fractional part of $x$. 


\section{ONE-TERM RECURSION FOR NONINTEGER $\rho$}

We consider the same class of functions as in the previous section, but we no longer require that $\rho$ be an integer. In our illustrations we take $\rho=2.1$. Figure 11 shows three examples. At the top, we take $f(x)=\cos \pi x$, so $F(x)$ is the Fourier transform of a Cantor measure; compare Figure 2, which comes from the same $f$ but different $\rho$. In the middle, $f(x)=1+2 \sin ^{2} \pi x$; compare Figure 3 (right). (Note, however, that the function for $\rho=2.1$ has no interpretation in the theory of wavelets.) In the bottom part of Figure 11 we go a step further, taking for $f(x)$ the function $\frac{1}{2}\left(\cos ^{2} x+\cos ^{2} \pi x\right)$, which is not periodic, but almost periodic.

In Figure 12 we show the graphs of successive $h_{n}$ for the function $F$ of Figure 11 (middle). This figure suggests that the conclusions of Proposition 2.1 and Corollary 2.2 should continue to hold in this case, but we no longer have the asymptotic selfsimilarity.

Proposition 3.1. The conclusions of Proposition 2.1 are valid for all real $\rho>1$.

Proof. The only difference in the proof is in the argument showing that the sequence $\rho^{j} x$ is uniformly distributed mod 1 for almost every $x$. This result is essentially contained in [Koksma 1935], but for the sake of completeness, we give the argument.

By a well-known criterion of Weyl, $\rho^{k} x$ is uniformly distributed mod 1 if and only if

$$
\lim _{n \rightarrow \infty} \frac{1}{n} \sum_{k=1}^{n} \exp \left(2 \pi i m \rho^{k} x\right)=0
$$

for every integer $m \neq 0$. Since we claim this for almost every $x$, we can without loss of generality set $m=1$. We first look at $L^{2}$ norms. Fix an interval $a \leq x \leq b$. Then

$$
\begin{aligned}
\int_{a}^{b}\left|\frac{1}{n} \sum_{k=1}^{n} \exp \left(2 \pi i \rho^{k} x\right)\right|^{2} d x \\
\quad=\sum_{j=1}^{n} \sum_{k=1}^{n} \frac{1}{n^{2}} \int_{a}^{b} \exp \left(2 \pi i\left(\rho^{j}-\rho^{k}\right) x\right) d x \\
\quad=\frac{b-a}{n}+\frac{2}{n^{2}} \sum_{1 \leq j<k \leq n} \int_{a}^{b} \cos 2 \pi\left(\rho^{j}-\rho^{k}\right) x d x \\
\quad \leq \frac{b-a}{n}+\frac{2}{\pi n^{2}} \sum_{1 \leq j<k \leq n} \frac{1}{\rho^{k}-\rho^{j}} \leq \frac{c}{n},
\end{aligned}
$$
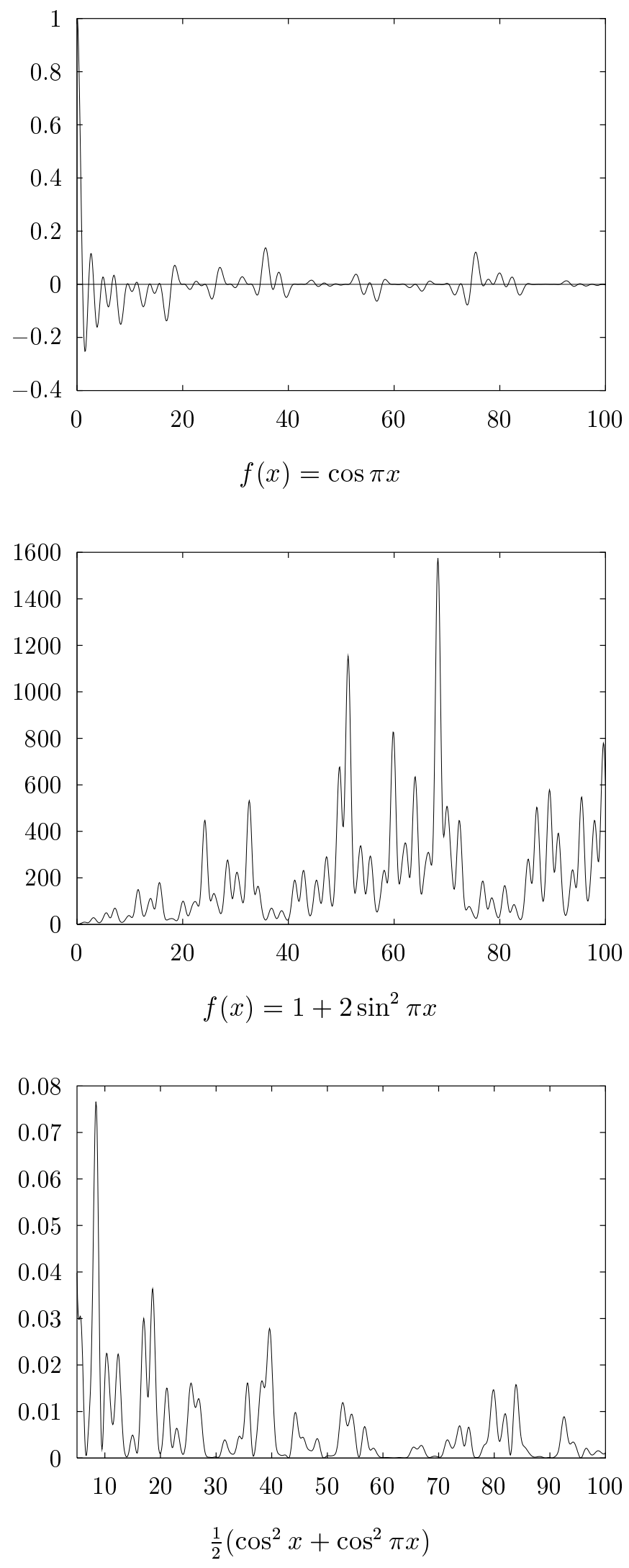

FIGURE 11. Graphs of $F(x)$ for $\rho=2.1$ and the indicated functions $f(x)$. 


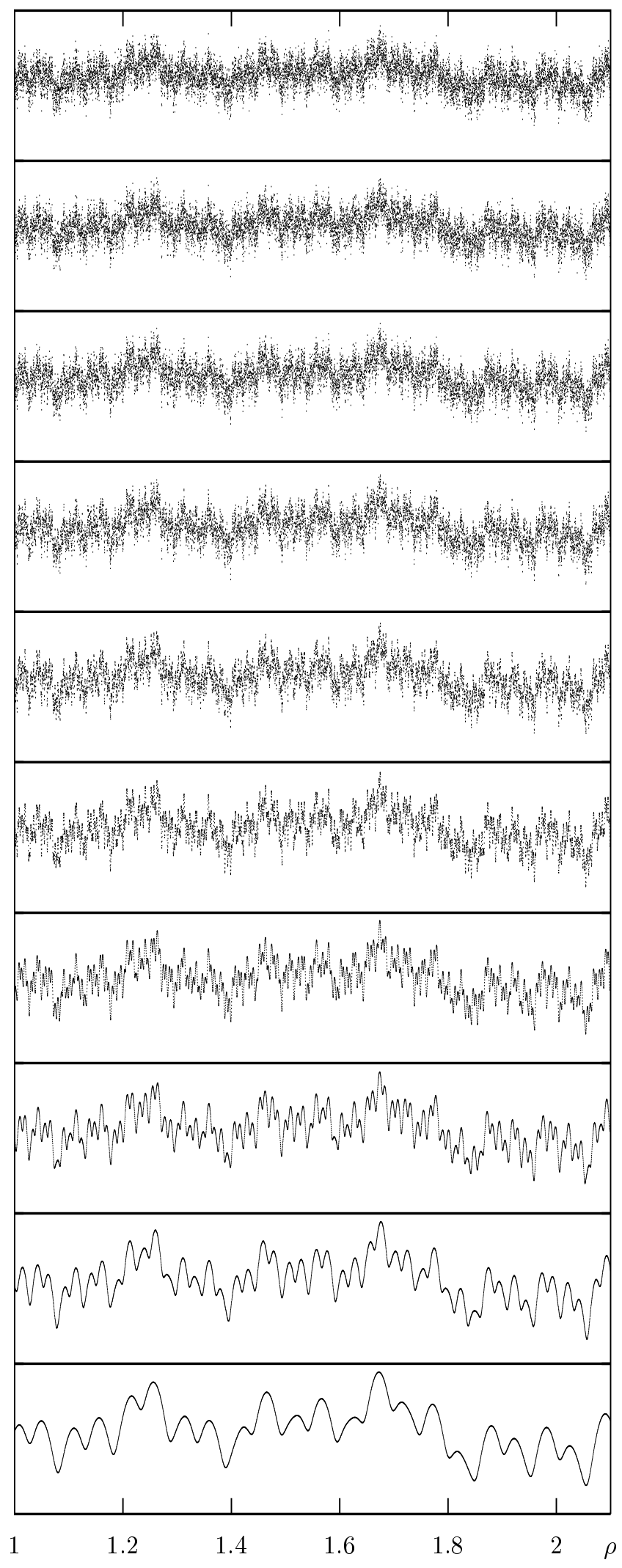

FIGURE 12. Graphs of $h_{n}(x)$, for $5 \leq n \leq 14$, for the function $F$ arising from $f(x)=1+2 \sin ^{2} \pi x$ and $\rho=2.1$. since

$$
\frac{1}{\rho^{k}-\rho^{j}}=\frac{1}{\rho^{k-j}-1} \frac{1}{\rho^{j}} \leq \frac{1}{\rho-1} \frac{1}{\rho^{j}}
$$

and $\sum_{j=1}^{\infty} 1 / \rho^{j}<\infty$. Thus, if we write $s_{n}(x)=$ $\frac{1}{n} \sum_{k=1}^{n} \exp \left(2 \pi i \rho^{k} x\right)$, we have

$$
\left\|s_{n}\right\|_{2}^{2} \leq \frac{c}{n},
$$

which implies $s_{n^{2}}(x) \rightarrow 0$ for almost every $x$. But a simple argument shows that $n^{2} \leq N \leq(n+1)^{2}$ implies

$$
\begin{aligned}
\mid s_{N}(x)- & s_{n^{2}}(x) \mid \\
& =\left|\left(\frac{1}{N}-\frac{1}{n^{2}}\right) \sum_{1}^{n^{2}} e^{2 \pi i \rho^{k} x}+\frac{1}{N} \sum_{n^{2}+1}^{N} e^{2 \pi i \rho^{k} x}\right| \\
& \leq n^{2}\left(\frac{1}{n^{2}}-\frac{1}{N}\right)+\frac{1}{N}\left(N-n^{2}\right) \leq \frac{c}{n}
\end{aligned}
$$

so convergence of the subsequence implies convergence of the sequence.

Proposition 3.1 can be extended to multiperiodic functions on certain nilpotent Lie groups [Strichartz 1992a].

In Figure 13 we show the graphs of consecutive $h_{n}(x)$ for the function $F(x)$ in Figure 11 (bottom). Despite the fact that $f$ is only almost periodic, we see the same convergence.

Proposition 3.2. Suppose $f(x)$ is uniformly almost periodic and bounded away from zero, that is,

$$
f(x) \geq \varepsilon>0 \quad \text { for all } x .
$$

Then

$$
\lim _{n \rightarrow \infty} h_{n}(x)=M(\log f)
$$

almost everywhere, where $M(\log f)$ denotes the socalled Bohr mean

$$
M(\log f)=\lim _{T \rightarrow \infty} \frac{1}{2 T} \int_{-T}^{T} \log f(t) d t .
$$

Proof. Because of (3.2), the function $\log f$ is also uniformly almost periodic. Therefore there exists a countable spectrum $\lambda_{0}=0, \lambda_{1}, \lambda_{2}, \ldots$ of reals such that $\log f(x)$ is the uniform limit of trigonometric polynomials of the form

$$
a_{0}+\sum_{j=1}^{N} a_{j} \exp \left(2 \pi i \lambda_{j} x\right),
$$




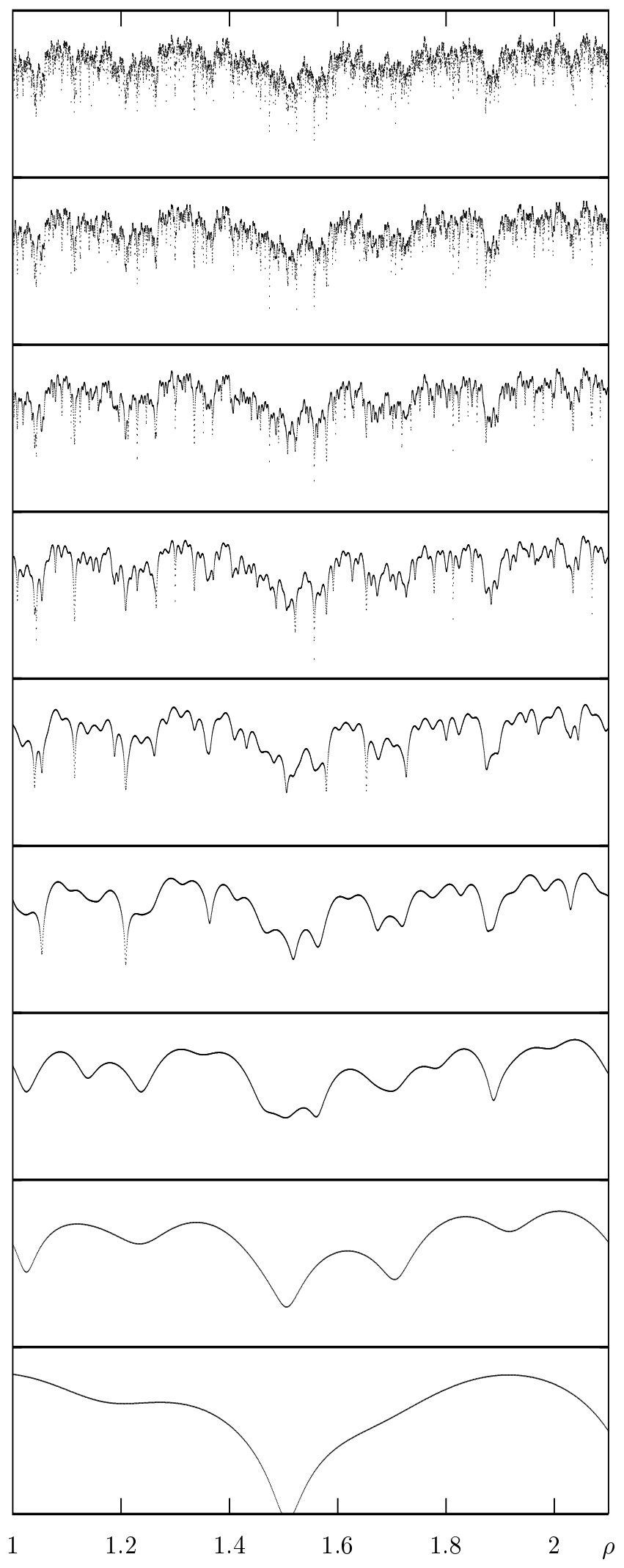

FIGURE 13. Graphs of $h_{n}(x)$, for $2 \leq n \leq 10$, for $F$ arising from $f(x)=\frac{1}{2}\left(\cos ^{2} x+\cos ^{2} \pi x\right)$ and $\rho=2.1$. Large negative values of $h_{n}(x)$ have been excised. where we may take $a_{0}=M(\log f)$. Since we can replace $\log f$ by this approximating sum with uniformly small error, to establish (3.3) almost everywhere it suffices to show that

$$
\lim _{n \rightarrow \infty} \frac{1}{n} \sum_{k=0}^{n-1} \exp \left(2 \pi i \lambda_{j} \rho^{k} x\right)=0
$$

for almost every $x$. Since there are only a countable number of $\lambda_{j}$, we are back to proving (3.1), which we have already done.

There is also a statement analogous to Corollary 2.2, where we drop the assumption (3.2) and assume only that $f$ is nonnegative and $\log f$ belongs to the Besicovitch class $B^{p}$ of almost periodic functions [Besicovitch 1954]. Then the limit (3.3) holds in the $B^{p}$ norm.

Figure 14 graphs the cumulative distribution of values of $h_{n}$ for $\rho=2.1$ and $f(x)=1+\sin ^{2} \pi x$. It is the analog of Figure 8 with $\rho$ changed to 2.1, and it shows a similar behavior.

\section{TWO-TERM RECURSION: THE COMMENSURABLE CASE}

In this section we study functions determined by the two-term relation

$$
F(x)=f_{1}\left(\frac{x}{\rho_{1}}\right) F\left(\frac{x}{\rho_{1}}\right)+f_{2}\left(\frac{x}{\rho_{2}}\right) F\left(\frac{x}{\rho_{2}}\right),
$$

where $\rho_{1}$ and $\rho_{2}$ are exponentially commensurable. To be specific, we take $\rho_{1}=2$ and $\rho_{2}=4$, but we expect the general case to be similar. We can rewrite (4.1) as

$$
F(4 x)=f_{1}(2 x) F(2 x)+f_{2}(x) F(x),
$$

and then, by induction,

$$
F\left(2^{n} x\right)=G_{n}(x) F(2 x)+H_{n}(x) F(x),
$$

where $G_{n}$ and $H_{n}$ satisfy the same recurrence relation,

$$
\begin{aligned}
& G_{n+1}(x)=f_{1}\left(2^{n} x\right) G_{n}(x)+f_{2}\left(2^{n-1} x\right) G_{n-1}(x), \\
& H_{n+1}(x)=f_{1}\left(2^{n} x\right) H_{n}(x)+f_{2}\left(2^{n-1} x\right) H_{n-1}(x),
\end{aligned}
$$

but with different initial conditions:

$$
\begin{array}{ll}
G_{0}(x)=0, & G_{1}(x)=1, \\
H_{0}(x)=1, & H_{1}(x)=0 .
\end{array}
$$




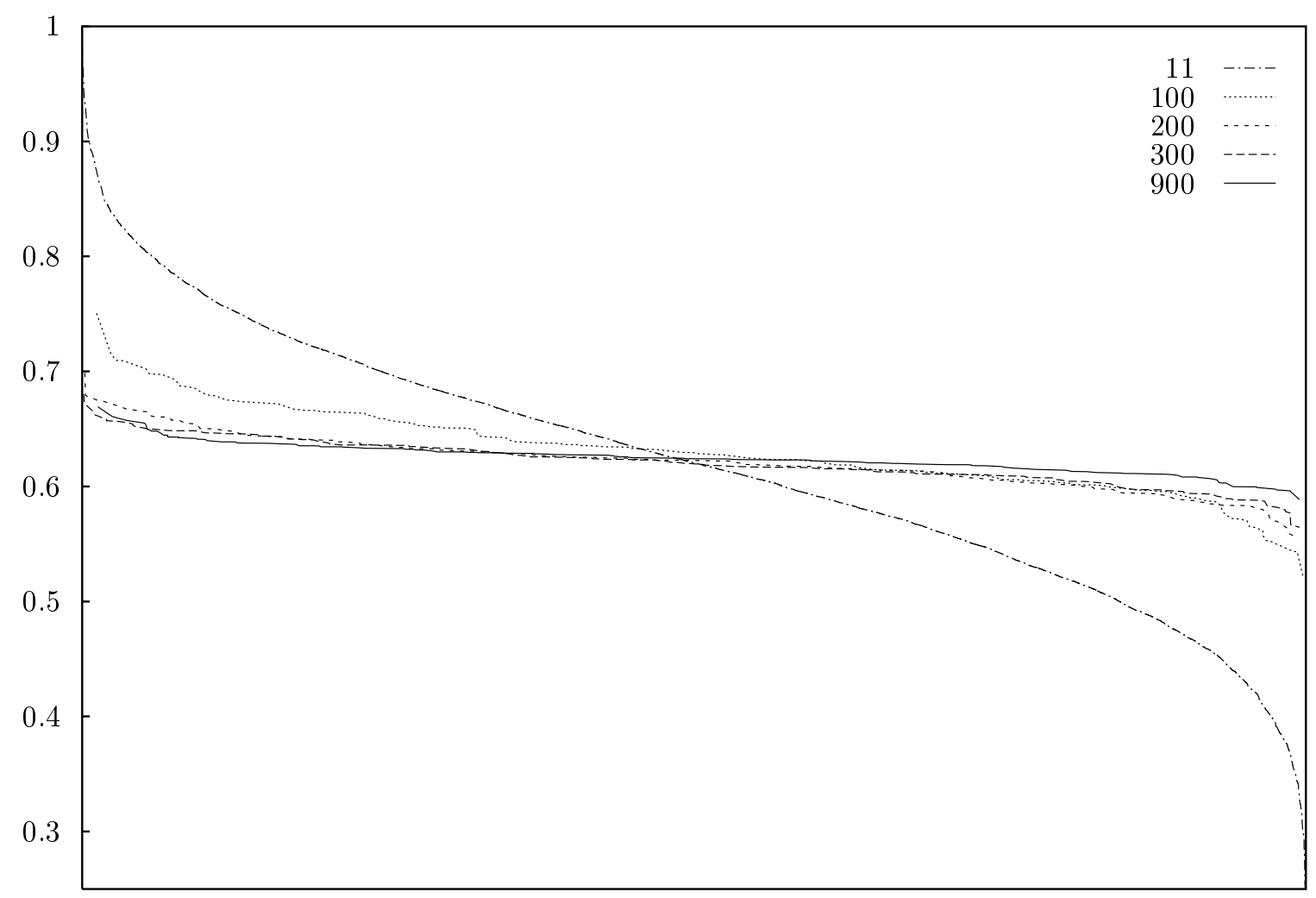

FIGURE 14. Cumulative distribution of values of $h_{n}(x)$ for the function $F$ of Figure 11 (middle) in the interval $1 \leq x \leq 2.1=\rho$. The functions were sampled at $2^{13}$ regularly spaced points.

All this follows easily by substituting (4.3) in (4.2) and equating factors of $F(2 x)$ and $F(x)$. We can also write the recurrence in matrix form:

$$
\left(\begin{array}{c}
G_{n+1}(x) \\
G_{n}(x)
\end{array}\right)=\left(\begin{array}{cc}
f_{1}\left(2^{n} x\right) & f_{2}\left(2^{n-1} x\right) \\
1 & 0
\end{array}\right)\left(\begin{array}{c}
G_{n}(x) \\
G_{n-1}(x)
\end{array}\right)
$$

and likewise for the $H_{n}$. If we set

$$
M(x)=\left(\begin{array}{cc}
f_{1}(2 x) & f_{2}(x) \\
1 & 0
\end{array}\right),
$$

it follows from (4.4) and (4.5) that

$$
\begin{aligned}
& G_{n}(x)=\left(M\left(2^{n-1} x\right) M\left(2^{n-2} x\right) \ldots M(x)\right)_{21}, \\
& H_{n}(x)=\left(M\left(2^{n-1} x\right) M\left(2^{n-2} x\right) \ldots M(x)\right)_{22},
\end{aligned}
$$

where the subscripts indicate matrix entries.

It follows from the theory of products of random matrices [Furstenberg and Kesten 1960] that there are positive constants $G$ and $H$ such that

$$
\lim _{n \rightarrow \infty} \frac{1}{n} \log G_{n}(x)=G \text { and } \lim _{n \rightarrow \infty} \frac{1}{n} \log H_{n}(x)=H
$$

almost everywhere.
If $G>H$, the first term predominates in (4.3), whereas if $G<H$, the second term predominates. Therefore

$$
\lim \frac{1}{n} \log F\left(2^{n} x\right)=\max (G, H)
$$

almost everywhere. We obtain the same conclusion if $G=H$. Thus we expect the graphs of

$$
\frac{1}{n} \log F\left(2^{n} x\right), \quad \frac{1}{n} \log G_{n}(x) \text { and } \frac{1}{n} \log H_{n}(x)
$$

all to follow the same pattern as the graphs of $h_{n}(x)$ in the one-term case.

Figure 15 shows the graph of $F(x)$ for

$$
\begin{aligned}
& f_{1}(x)=\frac{1}{2}+2 \sin ^{2} \pi x, \\
& f_{2}(x)=\frac{1}{2}+3 \sin ^{2} \pi x ;
\end{aligned}
$$

these functions are positive and satisfy the consistency condition

$$
f_{1}(0)+f_{2}(0)=1
$$

but otherwise have no special significance.

Figure 16 shows, for the same $F$, the graphs of $n^{-1} \log F\left(2^{n} x\right)$ for $10 \leq n \leq 14$. The oscillatory behavior of $n^{-1} \log G_{n}(x)$ and of $n^{-1} \log H_{n}(x)$ is 


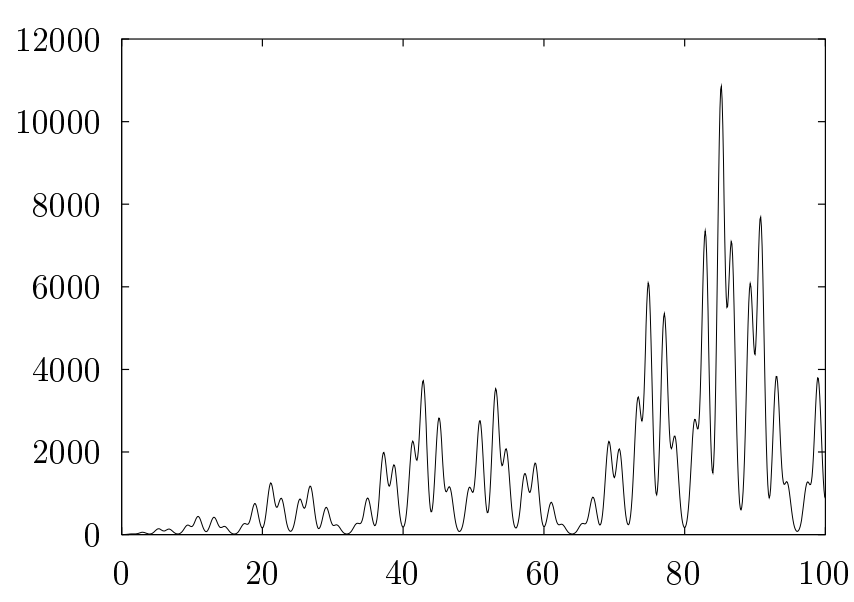

FIGURE 15. Graph of the solution $F(x)$ of (4.1), for $\rho_{1}=2, \rho_{2}=4, f_{1}(x)=.5+2 \sin ^{2} \pi x$, and $f_{2}(x)=.5+3 \sin ^{2} \pi x$.

the same as that of $n^{-1} \log F\left(2^{n} x\right)$; moreover, all three sequences show asymptotic self-similarity for the transformations $x \mapsto 2 x-1$ and $x \mapsto 2 x-2$. Figure 16 also graphs the differences

$$
\frac{1}{n}\left(\log F\left(2^{n} x\right)-\log G_{n}(x)\right)
$$

and

$$
\frac{1}{n}\left(\log F\left(2^{n} x\right)-\log H_{n}(x)\right) .
$$

Given this evidence, we are led to formulate the following conjecture:

Conjecture 4.1. Let $f_{1}$ and $f_{2}$ be positive continuous periodic functions satisfying (4.7), and let $\rho_{1}=\rho^{k_{1}}$ and $\rho_{2}=\rho^{k_{2}}$, where $k_{1}$ and $k_{2}$ are integers and $\rho>1$. If $F$ is as in (4.1), there exists a constant $C$ such that

$$
\lim _{n \rightarrow \infty} \frac{1}{n} \log F\left(\rho^{n} x\right)=C
$$

almost everywhere. Furthermore, if $\rho$ is an integer, the sequence is asymptotically self-similar for the transformations $x \mapsto \rho x-k$.

We conjecture that similar results hold for $m$-term generalizations of (4.1) and (4.7).

Problem 4.2. Find an interpretation for the constant in (4.8).

\section{TWO-TERM RECURSION: THE INCOMMENSURABLE CASE}

In this section we consider the same two-term recursion relation (4.1), but with $\rho_{1}$ and $\rho_{2}$ exponen-

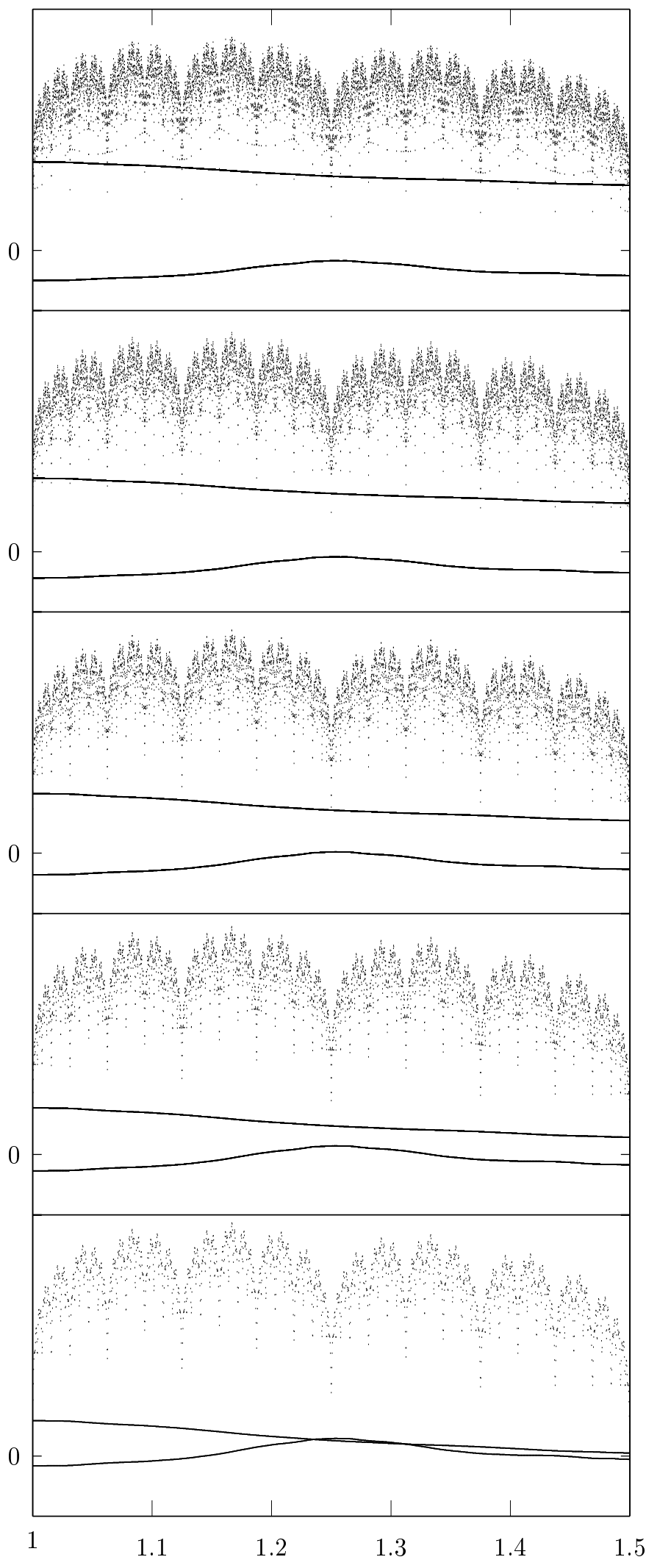

FIGURE 16. $F$ is as in Figure 15 and $n$ ranges from 10 to 14 as we go up. Within each unit, the jumpy graph is that of $n^{-1} \log F\left(2^{n} x\right)$; the smooth curves represent the differences $n^{-1}\left(\log F\left(2^{n} x\right)-G_{n}(x)\right)$ (upper) and $n^{-1}\left(\log F\left(2^{n} x\right)-H_{n}(x)\right)$ (lower). 
tially incommensurable. In Figure 17 we show the graph of $F$ for the choice

$$
\begin{aligned}
& \rho_{1}=2, \quad f_{1}(x)=.3+.2 \cos \pi x, \\
& \rho_{2}=3, \quad f_{2}(x)=.4+.1 \cos \pi x,
\end{aligned}
$$

first using a linear scale and then a log-log scale. The second graph seems to suggest that the bulk of the data is following roughly a straight line, but we have not been able to quantify the effect, as we did by the use of the functions $h_{n}$ in the one-term recursion case.

In Figure 18 we show the graph of $F$ for the choice

$$
\begin{array}{ll}
\rho_{1}=3, & f_{1}(x)=\frac{1}{2}, \\
\rho_{2}=4, & f_{2}(x)=\frac{1}{2} \cos \pi x .
\end{array}
$$

In this case, $F$ is the Fourier transform of a Cantortype measure on the interval $\left[-\frac{1}{3}, \frac{1}{3}\right]$, constructed by iterating the following procedure: give the middle third of the interval probability $\frac{1}{2}$ and the outer two quarters of the interval probability $\frac{1}{4}$ each.

Since we were not able to find any regularity in the pointwise behavior of $F$, we considered the integrals

$$
I_{q} F(x)=\int_{0}^{x}|F(t)|^{q} d t
$$

(Note that here, in contrast to the one-term recursion case, the function $|F(x)|^{q}$ does not satisfy a recursion relation of the same form as (4.1).) From [Strichartz 1993] and [Lau], we know that, for the example of Figure 18,

$$
\lim _{x \rightarrow \infty} x^{\alpha-1} I_{2} F(x)=c,
$$

where $\alpha=1-b \approx 0.815867$ is the solution of

$$
\frac{1}{2^{2}} 3^{\alpha}+\frac{1}{4^{2}} 4^{\alpha}+\frac{1}{4^{2}} 4^{\alpha}=1 \text {. }
$$

However, there are no known results that predict the behavior of $I_{q} F$ for $q \neq 2$ for this example, or even $I_{2} F$ for the example of Figure 17. We decided to test the hypothesis

$$
\lim _{x \rightarrow \infty} x^{-b_{q}} I_{q} F(x)=c_{q},
$$

and estimate the constant $b_{q}$. Since (5.3) is equivalent to

$$
\lim _{x \rightarrow \infty} \log I_{q} F(x)-\left(b_{q} \log x+\log c_{q}\right)=0,
$$
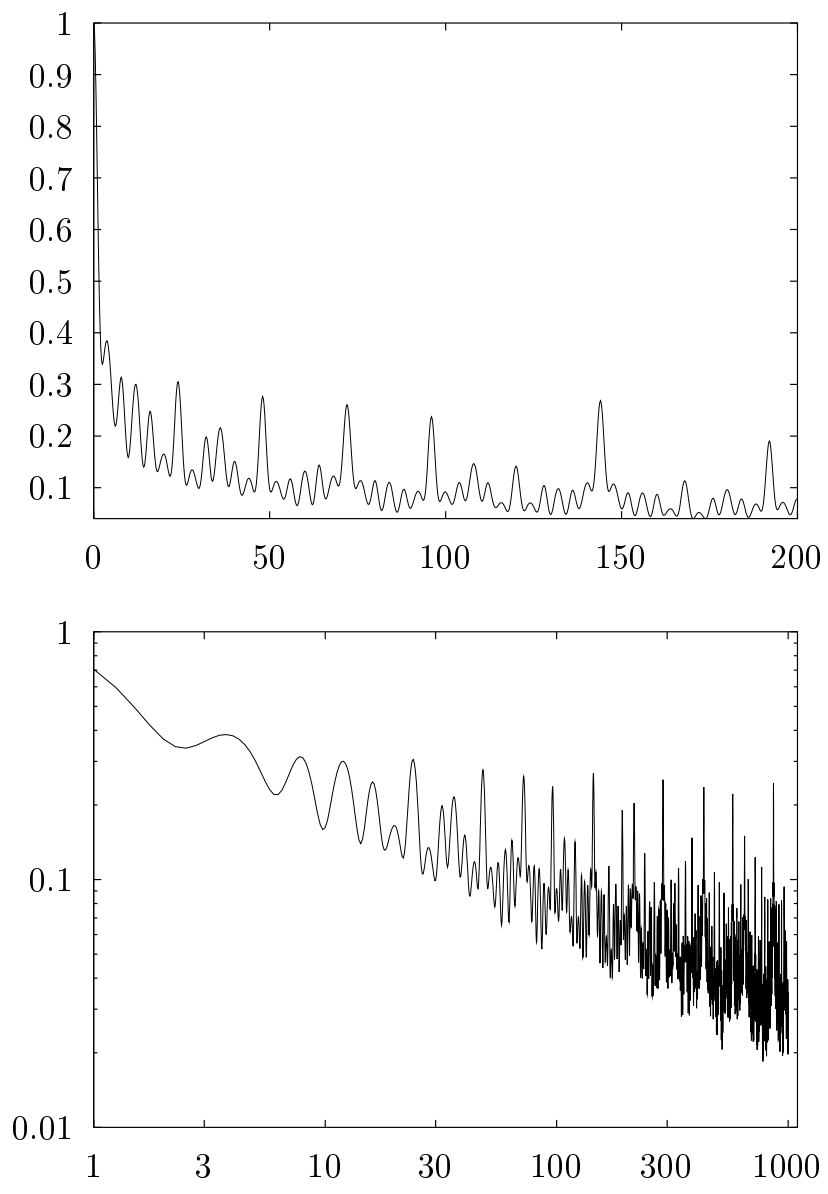

FIGURE 17. Graphs of the solution $F(x)$ of (4.1), for $\rho_{1}=2, \rho_{2}=3, f_{1}(x)=.3+.2 \cos \pi x$ and $f_{2}(x)=.4+.1 \cos \pi x$. The bottom graph uses a log-log scale.

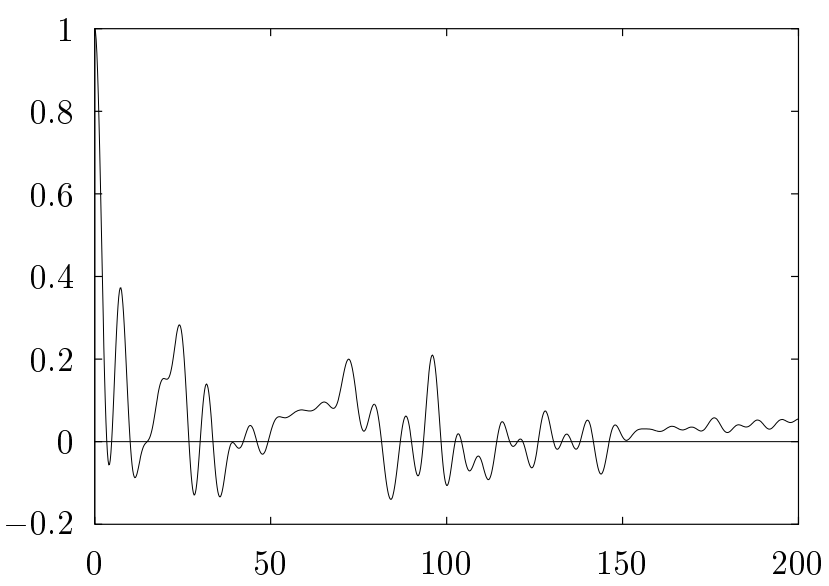

FIGURE 18. Graph of $F(x)$ for $\rho_{1}=3, \rho_{2}=4$, $f_{1}(x)=\frac{1}{2}$ and $f_{2}(x)=\frac{1}{2} \cos \pi x$. 
this involves plotting $I_{q} F$ on a log-log scale, noting the resemblance to a straight line for large values of $\log x$ (we did not perform statistical tests), and approximating $b_{q}$ by the slope of the least mean square best-fitting line.
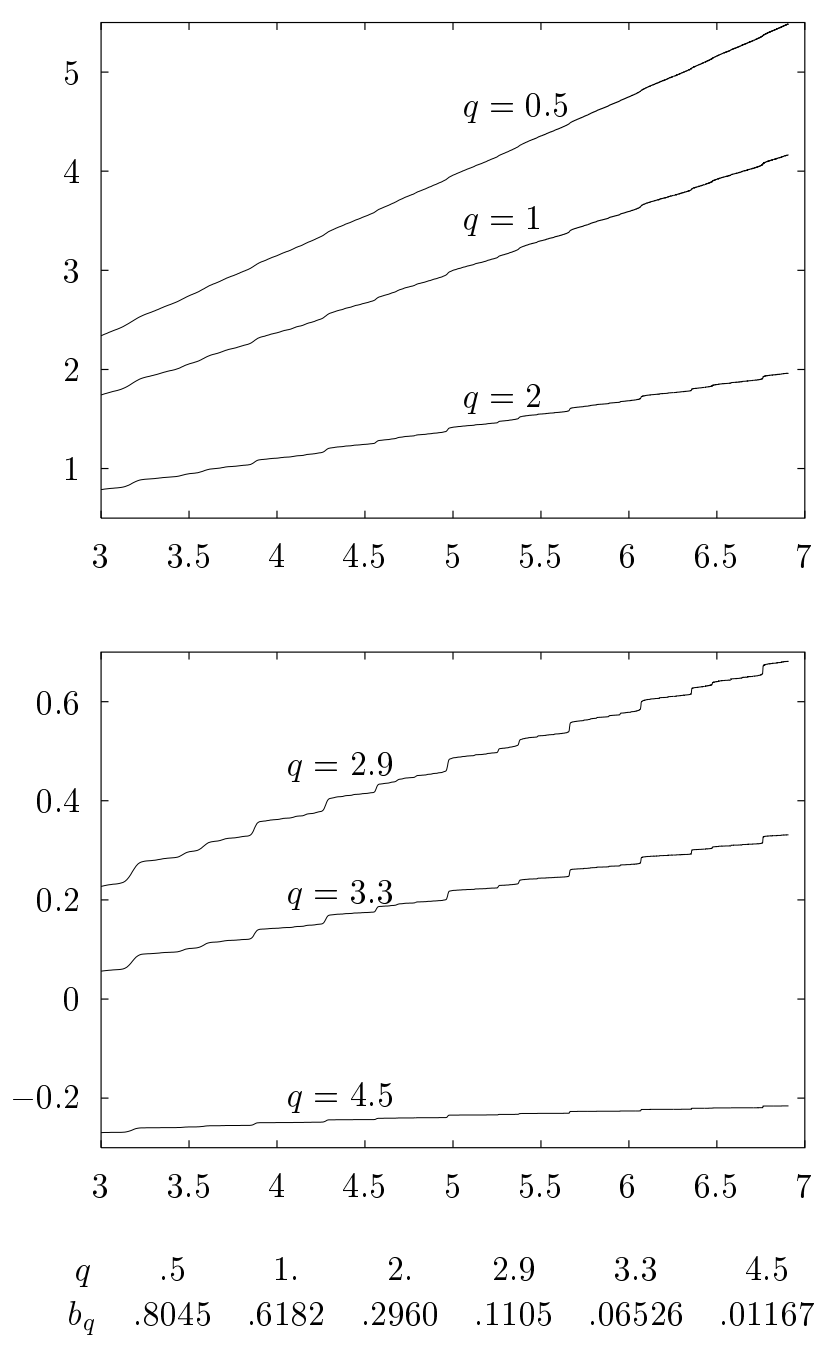

FIGURE 19. Graphs of $\log I_{q} F(x)$ as functions of $\log x$, for the function $F$ of Figure 17, and values of $b_{q}$ estimated therefrom.

Figure 19 shows the graph of $\log I_{q} F(x)$ versus $\log x$ for the function $F$ of Figure 17 for typical values of $q$. It is clear that the deviation from straightness becomes worse as $q$ increases. This is not surprising in view of the fact that the higher powers of $|F|$ tend to put more emphasis on values that deviate most from the general trend. Our evidence is certainly consistent with (5.4), but we would have liked to continue the computations out to larger values of $\log x$ in order to confirm the decrease in deviation from straightness. Unfortunately, this would involve computing integrals over very large intervals, and the accuracy of our data could not be relied upon.

As a different sort of check, we computed a second integral,

$$
I_{q}^{2} F(x)=\int_{0}^{x} I_{q} F(t) d t,
$$

for which we would expect

$$
\log I_{q}^{2} F(x)=\left(b_{q}+1\right) \log x+c_{q}^{\prime}
$$

with a greater accuracy of fit. In Figure 20 we show the graphs of $\log I_{q}^{2} F(x)$ versus $\log x$ for the three largest values of $q$ from Figure 19. It is clear that the desired smoothing has occurred, and the graphs are close to being straight, but the difference between the slopes of the best-fit lines for Figures 19 and 20 are not exactly equal to one, indicating the inaccuracy in our approximation to $b_{q}$ (assuming, of course, that (5.4) is correct).

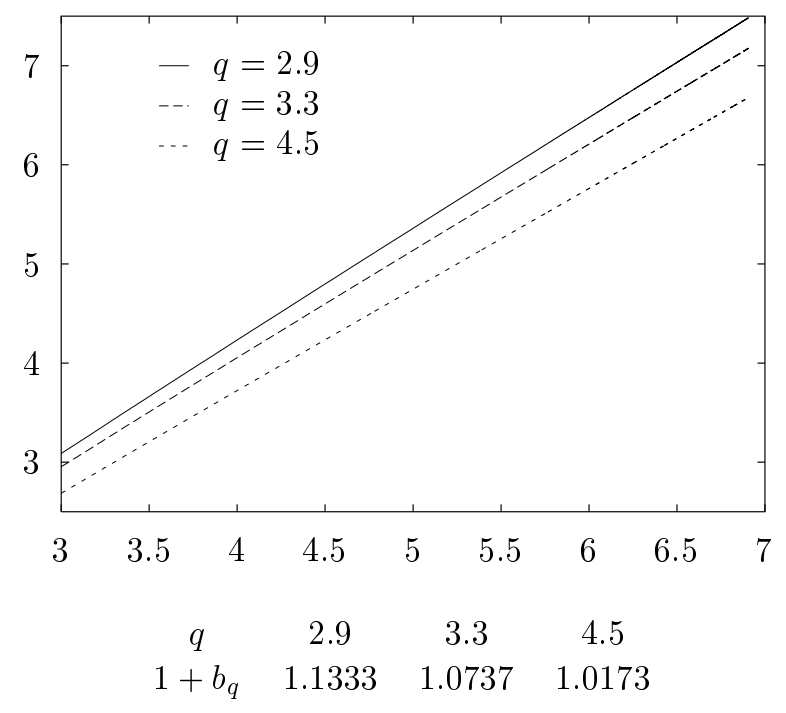

FIGURE 20. Graphs of $\log I_{q}^{2} F(x)$ as functions of $\log x$, for the function $F$ of Figure 17, and values of $1+b_{q}$ estimated therefrom.

In Figure 21 we plot the graph of $b_{q}$ as a function of $q$ for $.5 \leq q \leq 4.5$, as estimated by the slope of the best line fit to the computed data for $I_{q} F$, and the graph of $b_{q}+1$ as estimated from the computed data for $I_{q}^{2} F$.

Figures 22-24 show graphs analogous to those of Figures 19-21, for the function $F$ of Figure 18. Since this function displays greater variability than 


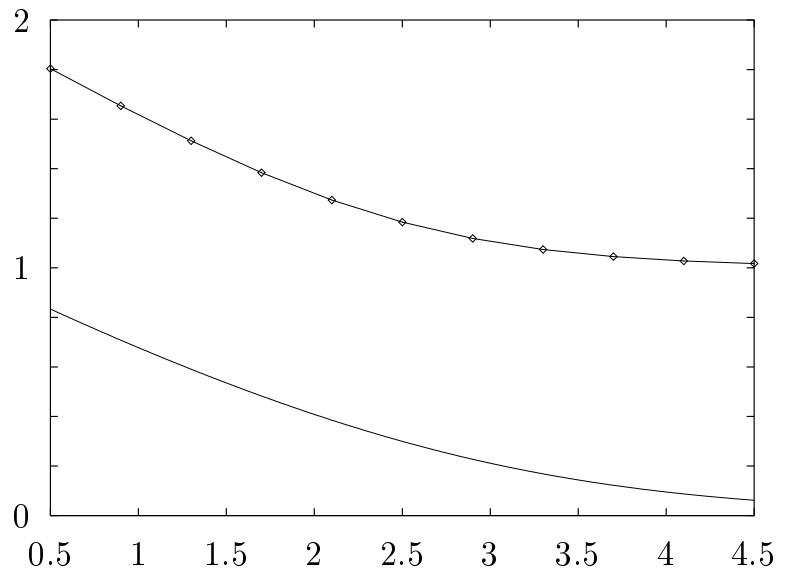

FIGURE 21. Graphs of $b_{q}$ as a function of $q$ (lower curve) and of $1+b_{q}$ as a function of $q$ (upper curve) for the function $F$ of Figure 17, as estimated from the slopes of $\log I_{q} F(x)$ and $\log I_{q}^{2} F(x)$ as functions of $\log x$. The step size for the first graph is .01 , and for the second graph .4 .

the first, we see greater deviations from straightness in the graphs of $\log I_{q} F(x)$. Note that the estimated value $b_{2} \approx .181399$ agrees well with the known value $1-\alpha$, where $\alpha$ is the root of (5.2).

Finally, in Figure 25, we graph the difference between $\log I_{2} F(x)$ versus $\log x$ and the best straightline fit for the function $F$ of Figure 18. There is a hint of a pattern here, but it is not yet clear exactly what it might be.

The experimental evidence seems to support a slightly weaker assertion than (5.3):

Conjecture 5.1. Let $f_{1}$ and $f_{2}$ be positive continuous periodic functions satisfying (1.4), let $\rho_{1}, \rho_{2}>1$, let $F$ satisfy (1.3), and define $I_{q} F$ by (5.1). Then

$$
b_{q}=\lim _{x \rightarrow 0} \frac{\log I_{q} F(x)}{\log x}
$$

exists for any $q>0$.

We conjecture that similar results hold for $m$-term generalizations of (1.3).

Problem 5.2. Find an interpretation for $b_{q}$.

\section{JULIA SETS}

In this section we let $F(x)$ be the Fourier transform of the equilibrium measure on a Julia set. For the quadratic polynomial

$$
f_{r}(z)=z^{2}-r,
$$
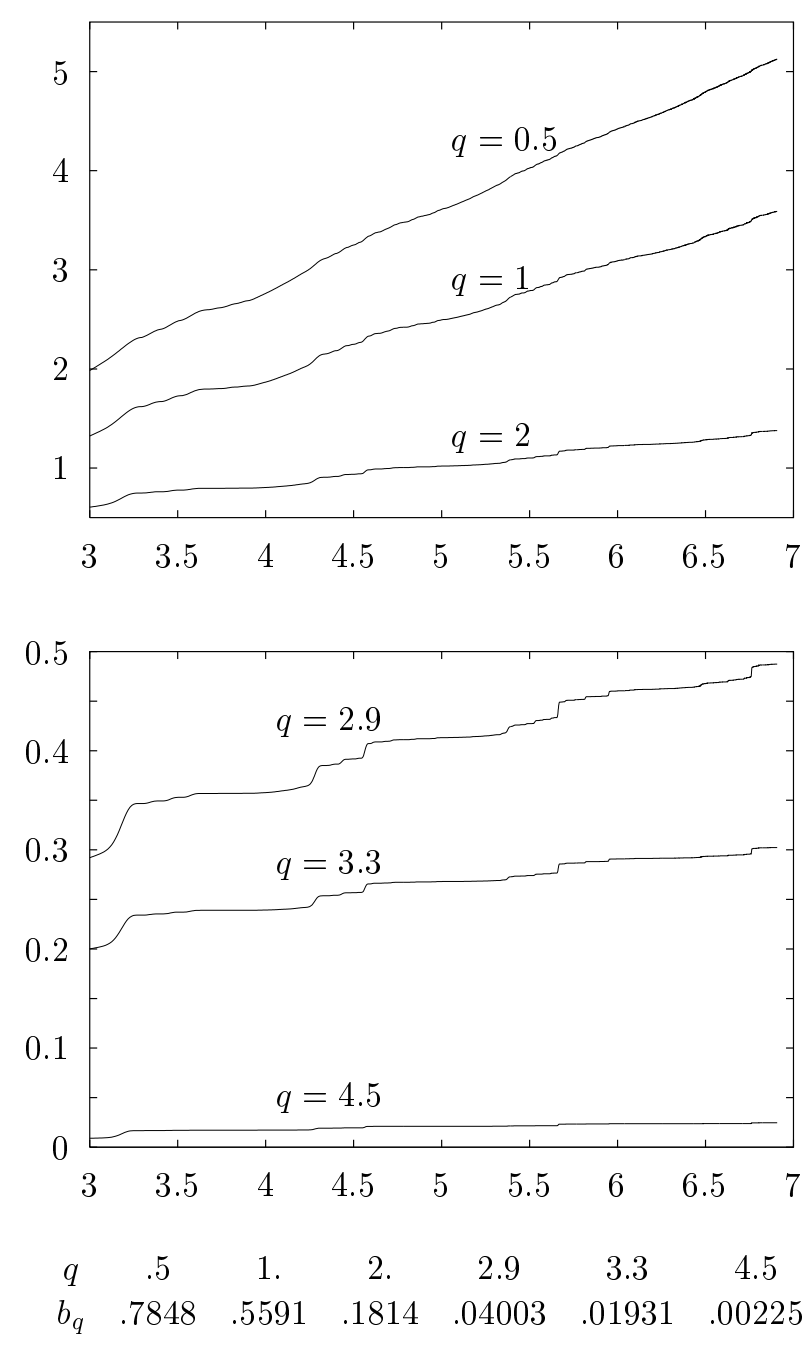

FIGURE 22. Graphs of $\log I_{q} F(x)$ as functions of $\log x$, for the function $F$ of Figure 18, and values of $b_{q}$ estimated therefrom.

with $r>2$, it is known that the Julia set $J_{r}$ is a totally disconnected (Cantor) set, contained in the real line. Figure 26 shows $J_{2.1}$.

Let

$$
\varphi_{r}^{ \pm}(x)= \pm \sqrt{x+r}
$$

denote the two inverse images of $x$ under $f_{r}$, where $x>-r$. This defines two homeomorphisms $\varphi_{r}^{+}$and $\varphi_{r}^{-}$from $(-r, \infty)$ to $\pm(0, \infty)$. There is a unique probability measure $\mu_{r}$, supported on $J_{r}$, that satisfies

$$
\mu_{r}(E)=\frac{1}{2} \mu_{r}\left(\varphi_{r}^{+}(E)\right)+\frac{1}{2} \mu_{r}\left(\varphi_{r}^{-}(E)\right) .
$$

This measure is easily approximated by randomly applying the mappings $\varphi^{ \pm}$. Because $\mu_{r}$ is symmet- 


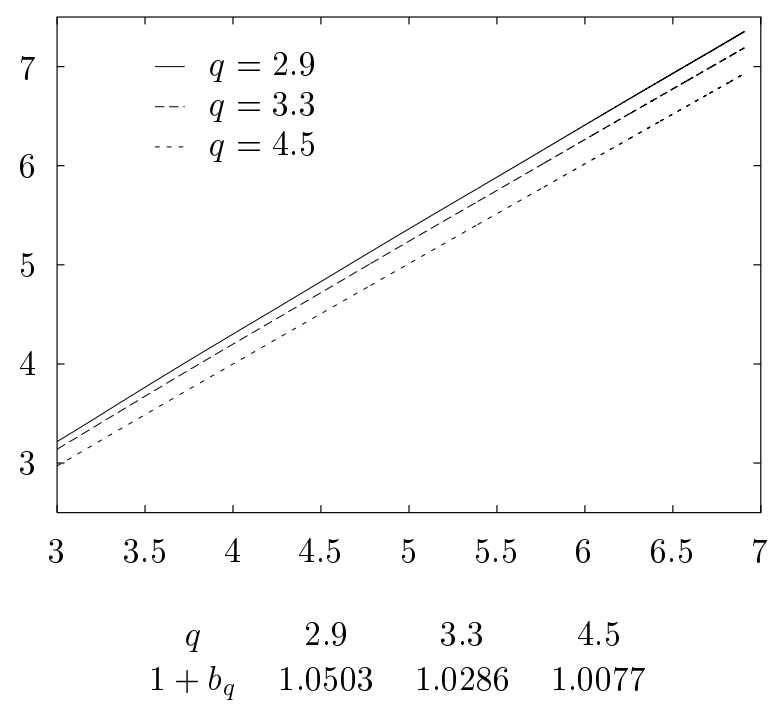

FIGURE 23. Graphs of $\log I_{q}^{2} F(x)$ as functions of $\log x$, for the function $F$ of Figure 18, and values of $1+b_{q}$ estimated therefrom.

ric about the origin, the Fourier transform is just a cosine transform,

$$
F(x)=\int \cos (x y) d \mu_{r}(y)
$$

which is approximated by

$$
F(x) \approx \frac{1}{N} \sum_{k=1}^{N} \cos \left(x y_{k}\right),
$$

for a sequence $y_{k} \in J_{r}$ defined recursively as follows:

$$
y_{k+1}=\varphi_{r}^{ \pm}\left(y_{k}\right),
$$

with \pm chosen randomly with equal probability; the initial $y_{1}$ is obtained by starting with one of the points $\frac{1}{2} \pm \frac{1}{2} \sqrt{r^{2}+1}$, which are known to be on the Julia set, and randomly iterating $\varphi_{r}^{ \pm}$a few times. Of course, the larger $x$ is, the more points $N$ are needed for a good approximation.

We worked with two values of $r$ in our experiments: 2.1 and 6 . Figure 27 shows two portions of the graph of $F$ in each case. (See Section 7 for a discussion of the accuracy of these graphs.)

Figure 28 shows the graph of $I_{q} F$ on a log-log scale for $r=2.1$ and 6 , and $q=.5,1$ and 2. We

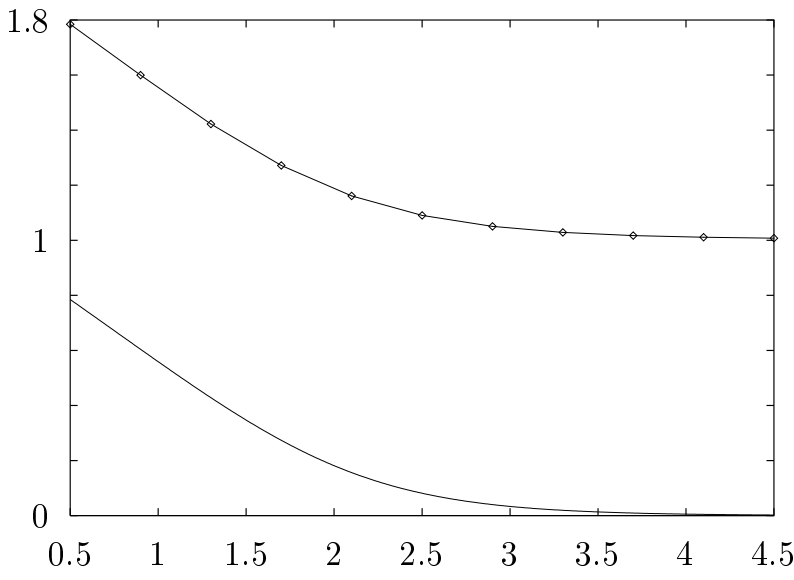

FIGURE 24. Graphs of $b_{q}$ as a function of $q$ (lower curve) and of $1+b_{q}$ as a function of $q$ (upper curve) for the function $F$ of Figure 17, as estimated from the slopes of $\log I_{q} F(x)$ and $\log I_{q}^{2} F(x)$ as functions of $\log x$. The step size for the first graph is .01 , and for the second graph .4 .

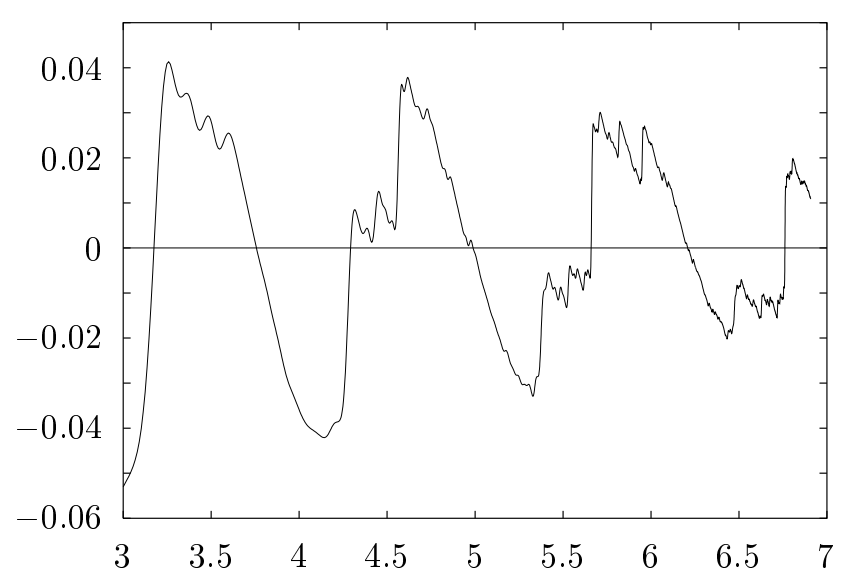

FIGURE 25. Difference between $\log I_{2} F(x)$ and the straight-line approximation $.1814 \log x-.1142$ as a function of $\log x$, for the function $F$ of Figure 18 .

computed the graphs for higher values of $q$, but they differ so much from a straight line that we hesitate to claim any significance for them.

Figure 29 plots the approximate slope as a function of $q$ for both values of $r$; the values of $q$ greater than 2 are included but are unlikely to be very accurate. The resemblance of these graphs to the corresonding graphs in Section 5 leads us to con-

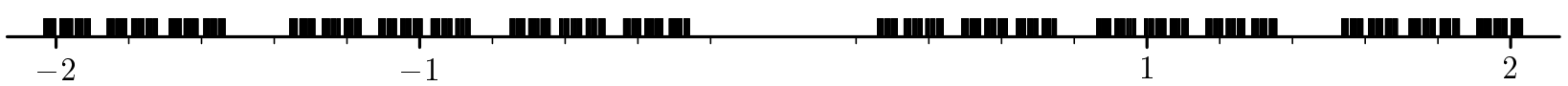

FIGURE 26. The Julia set $J_{2.1}$, dragged vertically for better visibility. 

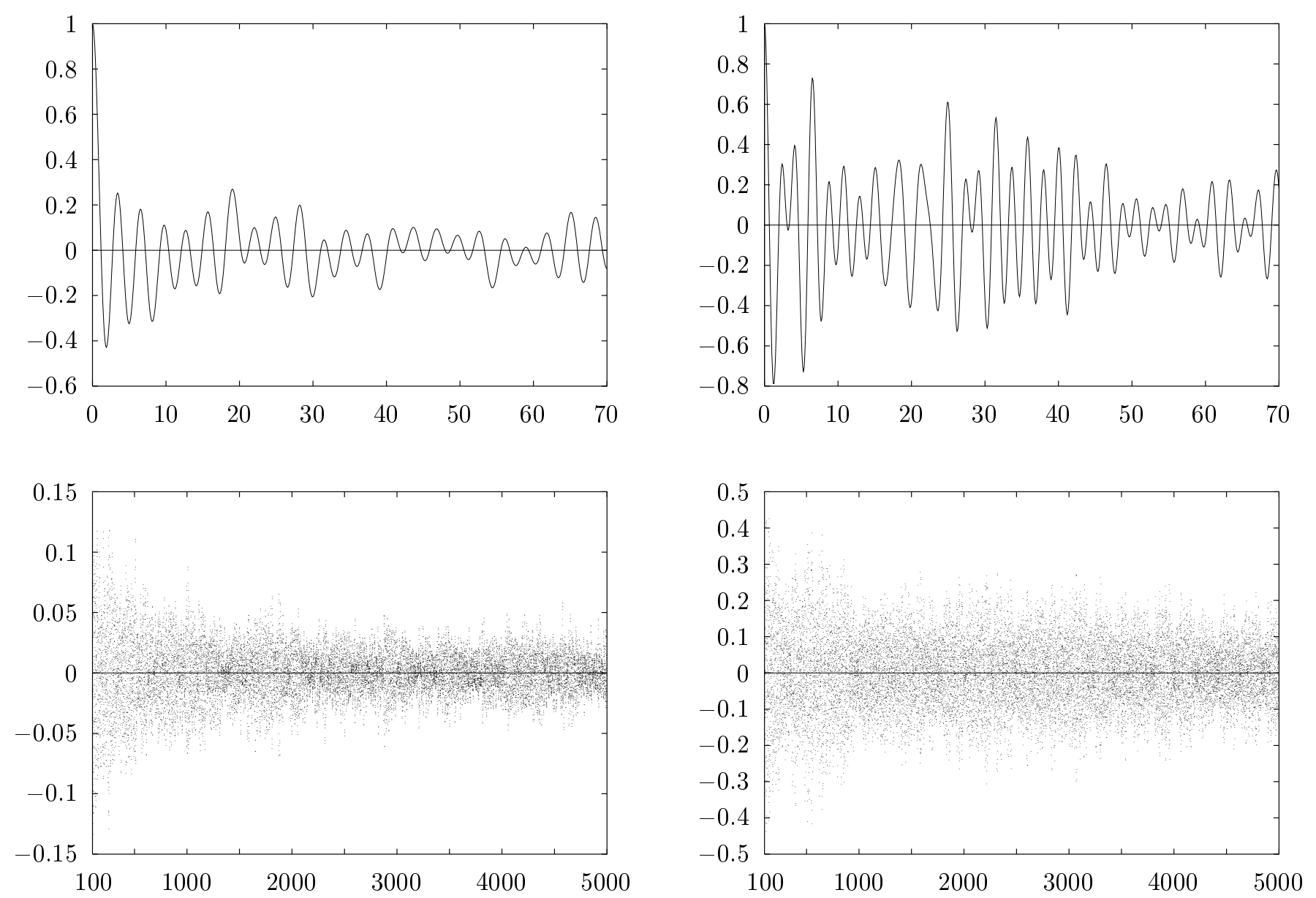

FIGURE 27. Graphs of $F=\hat{\mu}_{2.1}$ (left) and of $F=\hat{\mu}_{6}$ (right). To compute $F(x)$ we used (6.2), with $N=10,000$.
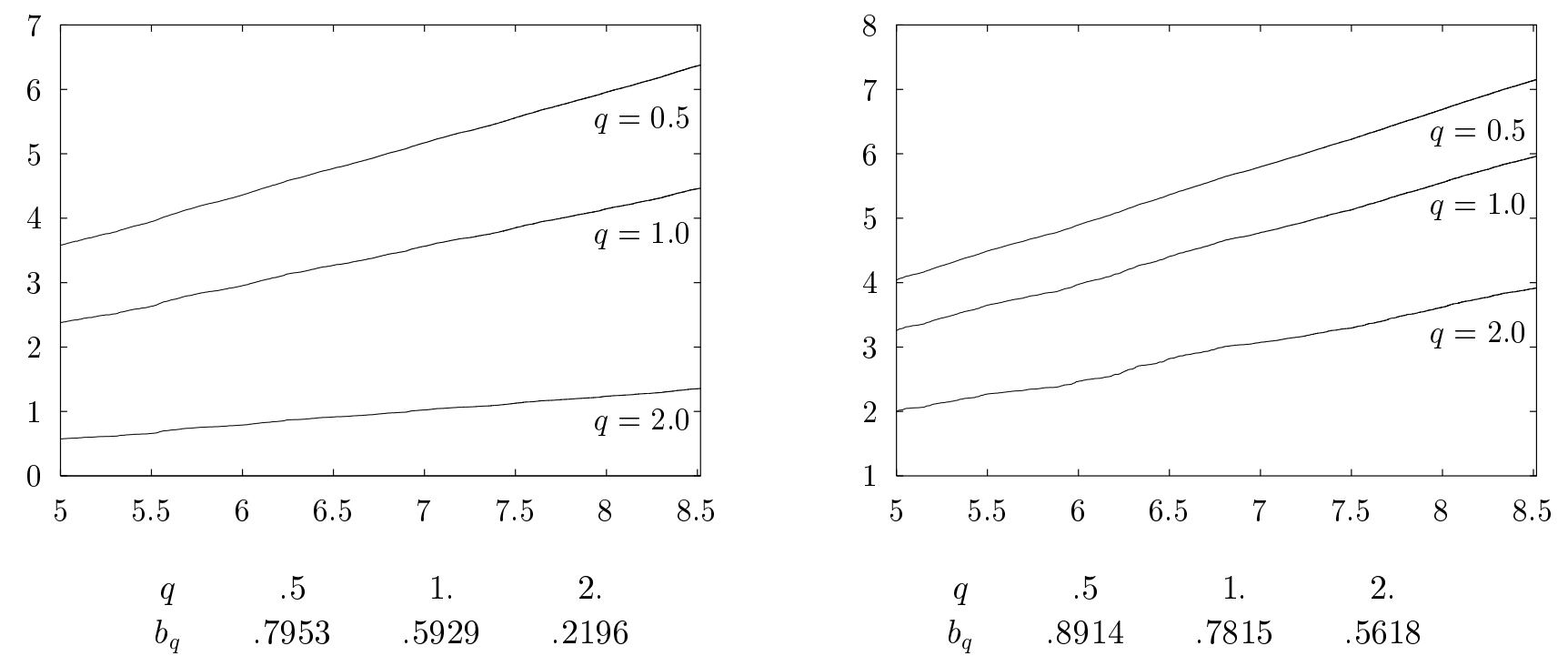

FIGURE 28. Graphs of $\log I_{q} F(x)$ as a function of $\log x$, for $F=\hat{\mu}_{2.1}$ (left) and $F=\hat{\mu}_{6}$ (right). 
jecture that (5.3) and (5.4) should hold, at least for $q \leq 2$.

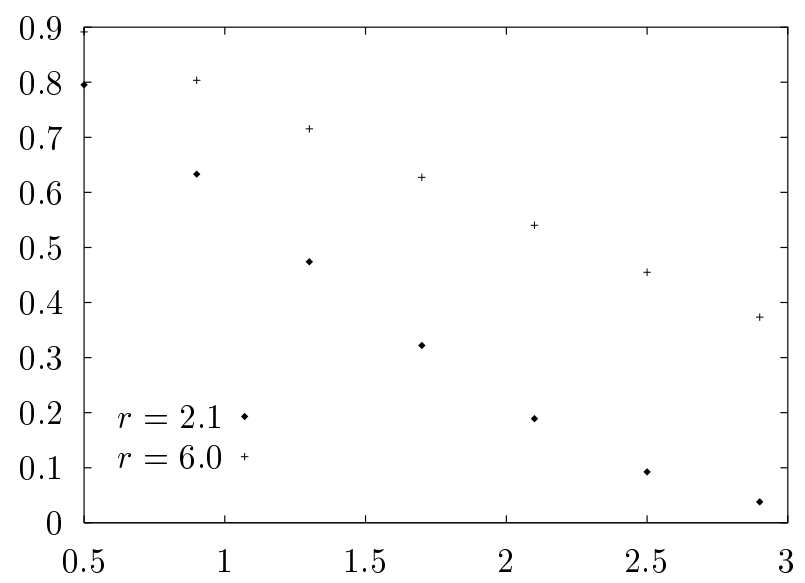

FIGURE 29. Values of $b_{q}$ as a function of $q$, for $F=\hat{\mu}_{2.1}$ and $F=\hat{\mu}_{6}$, as estimated from the slopes of $\log I_{q} F(x)$ as a function of $\log x$. The step size is 4 .

For $q=2$, the validity of (5.3) is equivalent, by a result of [Lau and Wang], to a more elementary statement in terms of the measure $\mu_{r}$. The mean quadratic variation of order $\alpha$ for a probability measure $\mu$ is defined to be

$$
\lim _{s \rightarrow 0} \frac{1}{s^{\alpha+1}} \int_{-\infty}^{\infty} \mu([x, x+s])^{2} d x
$$

if the limit exists. According to [Lau and Wang], this limit exists if and only if

$$
\lim _{x \rightarrow \infty} x^{\alpha-1} I_{2} F(x)
$$

exists for $F=\hat{\mu}$ (we assume for simplicity that $\mu$ is symmetric about the origin).

Now it is easier to compute the discrete analog of (6.4). Set

$$
\operatorname{MQV}(s)=\sum_{k=-\infty}^{\infty} \mu([k s,(k+1) s])^{2} .
$$

Then (6.4) is essentially equivalent to $\operatorname{MQV}(s) \approx$ $c s^{\alpha}$, or

$$
\log \operatorname{MQV}(s) \approx \alpha \log s+c^{\prime}
$$

We computed $\operatorname{MQV}(s)$ from (6.5), using the sequence $y_{k}$ generated by (6.3) to approximate the measure. The computation is made simpler by the fact that most of the intervals $[k s,(k+1) s]$ have measure zero. In Figure 30 we plot the data on a $\log -\log$ scale for both $r=2.1$ and $r=6$, along with a best straight-line fit. This gives strong support to the hypothesis (6.6), and the slope estimates $\alpha=.4392$ for $r=6$ and $\alpha=.7894$ for $r=2.1$ agree well with the predicted values of $1-b_{2}$ from Figure 28, namely .4382 and .7804.

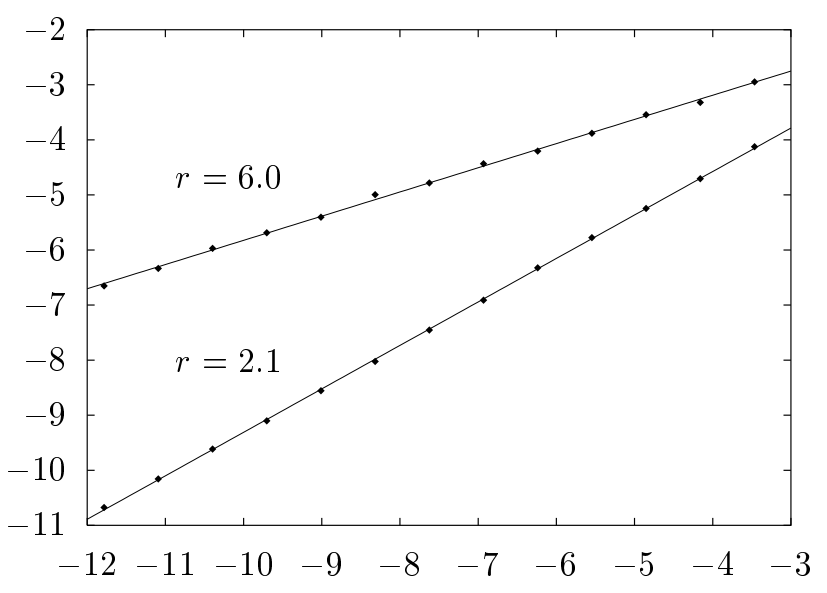

FIGURE 30. Values of $\log \mathrm{MQV}(s)$ as a function of $\log s$ (logarithms to base 2) for $F=\hat{\mu}_{2.1}$ and $F=\hat{\mu}_{6}$, together with the best-fitting straight line. The estimated slopes are .43923 for $r=6$ and .78945 for $r=2.1$.

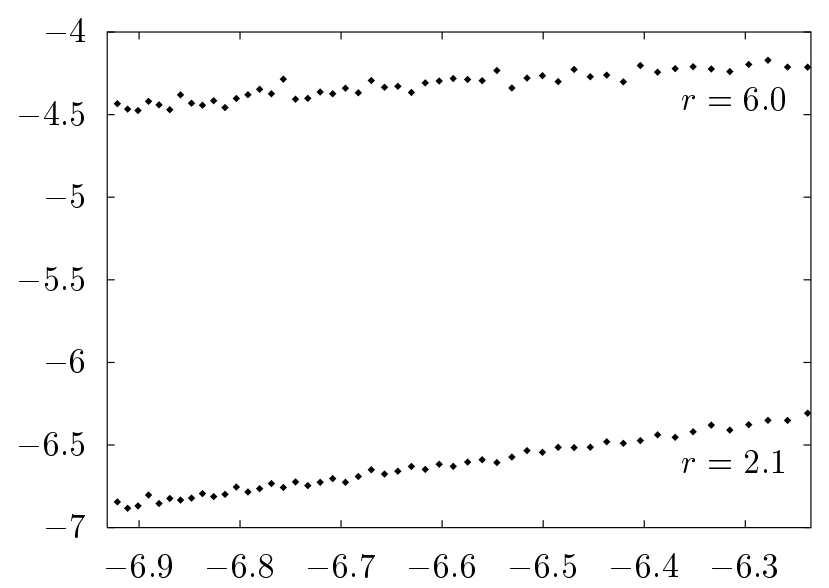

FIGURE 31. Values of $\log \mathrm{MQV}(s)$ as a function of $\log s$ (base 2) for $F=\hat{\mu}_{2.1}$ and $F=\hat{\mu}_{6}$ (detail).

However, the relative straightness of the log-log graphs of $\mathrm{MQV}(s)$ is misleading. In Figure 31 we fill in more detail, plotting $\operatorname{MQV}(s)$ on a log-log scale for more values of $s$ in a smaller range. The result shows much greater variability. In part, this might be accounted for by the discrete substitution of (6.5) for (6.4). (Although this involves the replacement of an integral by a Riemann sum of step size $s$, which tends to zero, the function being 
integrated also varies with $s$, so the best we can say is that

$$
\begin{aligned}
\frac{c_{1}}{s} \int_{-\infty}^{\infty} \mu([x, x+s])^{2} d x & \leq \operatorname{MQV}(s) \\
& \leq \frac{c_{2}}{s} \int_{-\infty}^{\infty} \mu([x, x+s])^{2} d x
\end{aligned}
$$

for certain constants $c_{1}$ and $c_{2}$.) But the variability could also be indicative that the limit (6.4) does not really exist, and that only some weaker conclusion holds, such as

$$
c_{1} \leq \frac{1}{s^{1+\alpha}} \int_{-\infty}^{\infty} \mu([x, x+s])^{2} d x \leq c_{2}
$$

for $0<s<1$. The order $\alpha$ in (6.7) represents some sort of dimension for the measure $\mu$, so it seems to be an interesting question to prove (6.7) for $\mu=\mu_{r}$ with $\alpha=\alpha_{r}$ given by some explicit or implicit function.

In [Strichartz 1992b] the weaker statement that

$$
\lim _{s \rightarrow 0} \frac{\log \int_{-\infty}^{\infty} \mu([x, x+s])^{p} d x}{\log s}
$$

exists is proved for $p>1$, and a procedure for computing the limit to any desired accuracy is given. In particular, this implies (1.9) for $q=2$.

\section{EXPERIMENTAL PROCEDURE}

In this section we explain briefly the computing methods used to produce the experimental results, and discuss questions of accuracy.

The experimental data in this paper were generated on the Sun SPARCserver 490 and SPARCstation 1 . The programs to generate the data were written in $\mathrm{C}$. The graphs were produced using Gnuplot (Unix version 2.0), with labels separately processed in $\mathrm{T}_{\mathrm{E}} \mathrm{X}$ at production time.

The primary program we wrote to generate the data takes as input the choice of a function, the range of $x$-values over which to observe the function, and the desired sampling rate. It outputs a file of $x$ - and $y$-values of the function, with a header containing information about the input data and program version, among other things. The program is written in a structured and flexible manner that facilitates the addition of new functions and modules at the user's discretion.
We also wrote auxiliary programs to further process precomputed function data, performing tasks such as integration by the Simpson rule, sorting by $x$ - or $y$-value, taking logs, and fitting a leastsquares straight line.

Our computations were carried out using the variable type "double", which in general proved sufficient. However, there were occasions when the error became appreciably large; this point will be discussed in more detail later.

When computing integrals, we had to keep in mind the error caused by the use of the Simpson rule. This could be minimized by sampling at as high a rate as feasible. In Figures 19-20, for example, the size of each $x$-interval is 0.25 .

When evaluating functions expressed as infinite sums or products, we had to come up with a reasonable finite cutoff. We chose $K \log x$, where the constant $K$ is chosen large enough that further increasing it did not affect the function values in the lower $x$-range. Multiplying by $\log x$ ensured that, as $x$ became larger, more terms of the infinite product or sum would be taken, as is desirable.

In spite of all precautions, the possibility was always present that the pictures we produced were slightly tainted by computer error. We checked for error whenever possible by repeating computations with some key parameter changed, and then checking to see if the computed values changed as expected.

In addition to the many computing obstacles that arose during the course of our research, other obstacles arising from the nature of the functions themselves often appeared, which we tried to solve in the best manner possible. Because we were studying the asymptotic, or long-range, behavior of the functions we were computing, it was often necessary to compute the logarithm of a function over a large $x$-range in order to better see how it was behaving. In fact, the logarithm was one of our most basic tools for interpreting our output in most cases. Of course, by the nature of the logarithm, we were forced to select functions that were nonnegative. However, many of the functions we wished to compute, for example, $f(x)=\cos x$, still had undesirable zeros even when converted to a nonnegative function by squaring.

We settled upon the following solution. The part of the program that scans the specified $x$-range and computes the log of the function value at each point 
along the way was modified to ignore $y$-values less than a user-controllable parameter $\varepsilon$. In this way, the program was able to weed out $y$-values too close to zero with a simple test. (Figures 4 and 13 show data so clipped.) Alternatively, the program could be set to compute the functions as usual, and the zero-test could be carried out in the helper program that computes logarithms of functions already in data-file format.

Another computing obstacle we faced, especially when trying to obtain data for very large $x$, in either the one- or two-term recursion cases, was that of compute time. This problem was dodged to some degree by the following change to the scanning algorithm: instead of sampling the $x$-range at regular steps, the modified program computed $y$ values at a specified number of points chosen randomly from the $x$-range. In this way, the program could produce a view of at least the bulk structure of the function without having to compute as many points. (The random-number generator used was called drand48(), and was seeded at each run with a long integer by the function srand48(). Both of these functions are part of the standard C library.) This method was most useful when combined with the fact that certain "average" behaviors were expected for certain types of functions at large values of $x$, such as the cumulative distributions in Figures 8 and 14. The random scan also came in handy a couple of times to clarify the behavior of some plots that looked the way they did only because of the regular step at which they were computed.

When $F(x)$ is defined by a recursion with two terms, the computational challenges are considerably greater than in the one-term case. How does one compute such a function? There is no explicit iterative formula for it, as there is in the one-term case. However, if one knows the value of $F(x)$ for some range of $x$, one can compute it for any other range by using the recursive formula. How does one calculate $F(x)$ for some range of $x$ ? We used a Taylor expansion about $x=0$.

In the formula for the $n$-th derivative of the function, we were able to group together all terms involving the $n$-th derivative of $F(x)$ to obtain an explicit formula for it in terms of its lower derivatives. Thus, using a recursive scheme, we were able to compute many of the derivatives of $F$ at 0 . We were then able to use those derivatives to construct a Taylor approximation for $F$ near zero. Once we had this approximation, we could use it to compute the value of $F(x)$ for any $x$ by employing recursion.

What about accuracy? As already mentioned, we used double precision, which gives 16 significant digits of accuracy. We used as many terms in the Taylor expansions as this accuracy would allow. We tested how far out these Taylor expansions could be trusted by looking to see how far out an increase in the number of Taylor terms failed to produce a detectable change in function values (see Figure 32). For the most part, we were able to trust Taylor expansions of 35 terms for $x$ between 0 and 0.5 at least.

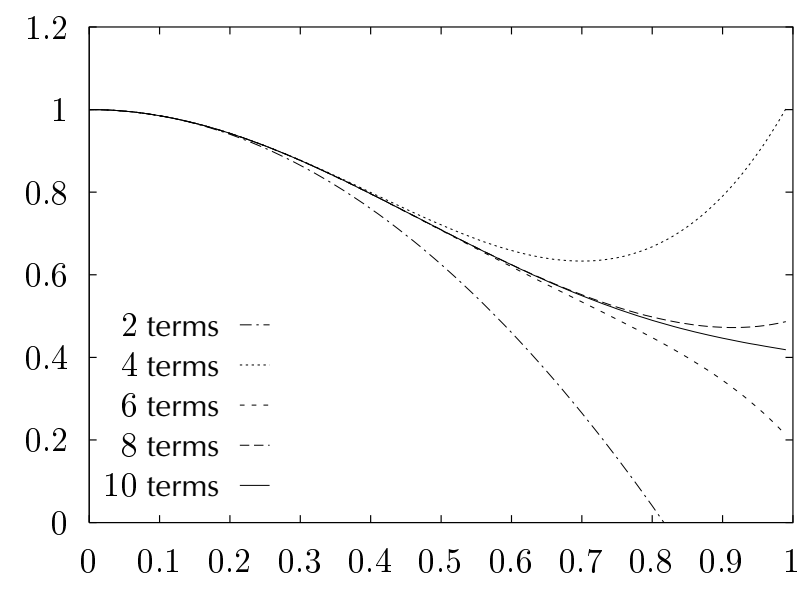

FIGURE 32. Graphs of the Taylor polynomial approximations to the function $F$ of Figure 15, with different cutoffs. The function $F$ is even, so only even powers of $x$ occur in the Taylor polynomials.

Of course, the above computational technique is not free from error. To obtain an idea of the size of the error, we examined the special case $\rho_{1}=2$ and $\rho_{2}=4$, where (4.3) holds. The computations of $G_{n}$ and $H_{n}$ from (4.6) are faster and more accurate, enabling us to compute $F(x)$ for larger values of $x$ with greater reliability. By comparing the output with the recursion-Taylor computation, we found the two in substantial agreement for $x$ between 0 and $10^{5}$.

The other time-consuming operation is the computation of the derivatives of $f$ at 0 . We can speed it up by storing the derivatives in an array as they are computed. When the next higher derivative needs to be computed, the algorithm need not recurse to do so, since all lower derivatives are already in memory. 
In spite of all this, the fact remains that twoterm recursion is computationally expensive, especially when the functions $f_{1}$ and $f_{2}$ are trigonometric functions or the like, and must be computed using time-consuming series expansions. If one of these functions is something computationally simple, like a constant or a polynomial of low degree, a lot of time can be saved.

More specific programming challenges and problems with accuracy arose in our investigations of the Fourier transforms of measures supported by Julia sets. We used the approximation (6.2). Immediately, we knew we might have trouble with the value for $N$ in that expression. The error in approximating the function in this way should be approximately $c N^{-1 / 2}$, where $c$ is a (hopefully small) constant. Thus, in order to approximate the function to a degree of accuracy of merely $\frac{1}{100}$, we already need 10,000 iterations for each $x$-value! If an interval is sampled for a scant 1000 points, we are talking about $10^{7}$ iterations, which is indeed an enormous amount of computation. To see just how good or bad things were, we looked at a small, randomly chosen, $x$-range for each value of $r$, and increased $N$ in steps of 1000 (later 10,000), to see what effect this had in the approximation. It was not until about $N=10,000$ that the qualitative appearance of the graphs of the function settled down to a constant shape. Even then, the deviation between two choices for $N$ was appreciable relative to the function values: see Figure 33. For practicality, we did stick with $N=10,000$. As can be seen in Figure 33, the error is not systematic, and thus we feel our computations are at least a

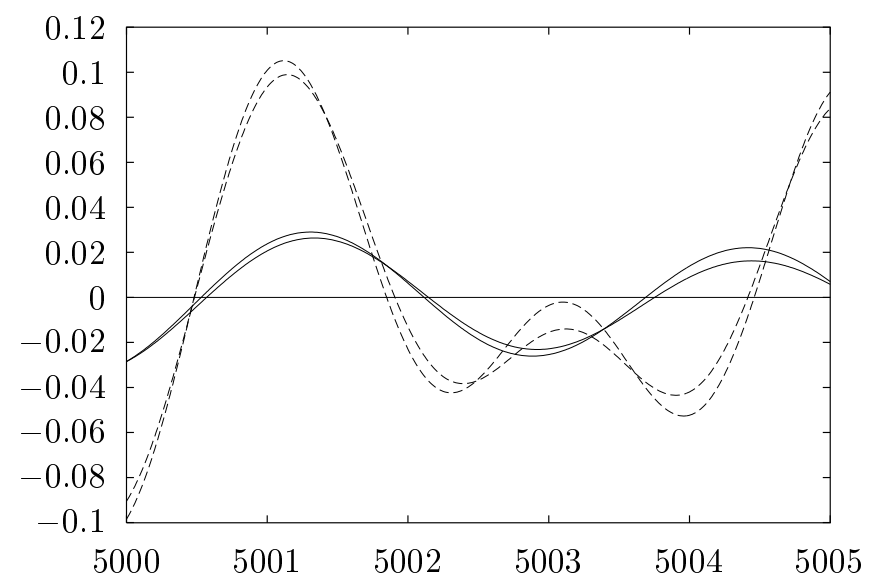

good beginning to the investigation of these Fourier transforms. Also note that certain values of $r$ lend themselves to better accuracy: for $r=6$, where the Julia set is much more concentrated than at $r=2.1$, there is some improvement in accuracy between the left and right part of the figure; whereas for $r=2.1$ there seems to be no improvement even with $N$ this high.

The other computation we performed with these Julia-set measures was a calculation of the mean quadratic variation (6.5), and we ran into problems of a similar nature.

For the first computation of $\operatorname{MQV}(s)$, we approximated the measure by "tossing" one hundred thousand points, and allowed $s$ to decrease from 1 down to $2^{-24}$. This produced the top set of points in Figure 34. What was happening here? Why was this very straight line bending as more $s$ values were added to the left? We conjectured that our interval size was getting so small, and thus the total number of slices considered so large, that this number was in fact much bigger than the 100,000 total points "thrown" in the first place. Since the function is a measure of probability, this could certainly account for erroneous computations as $s$ becomes very small. To test this, we generated as many points of the Julia set as we possibly could with our current memory capacity, and computed $\operatorname{MQV}(s)$ once more. This time (with $N=500,000)$, the new points of $\operatorname{MQV}(s)$ were indeed improved (again see Figure 34), although even with $N=500,000$ we are still nowhere near high enough to compute $\mathrm{MQV}(s)$ for $s$ to much less than $2^{-18}$.

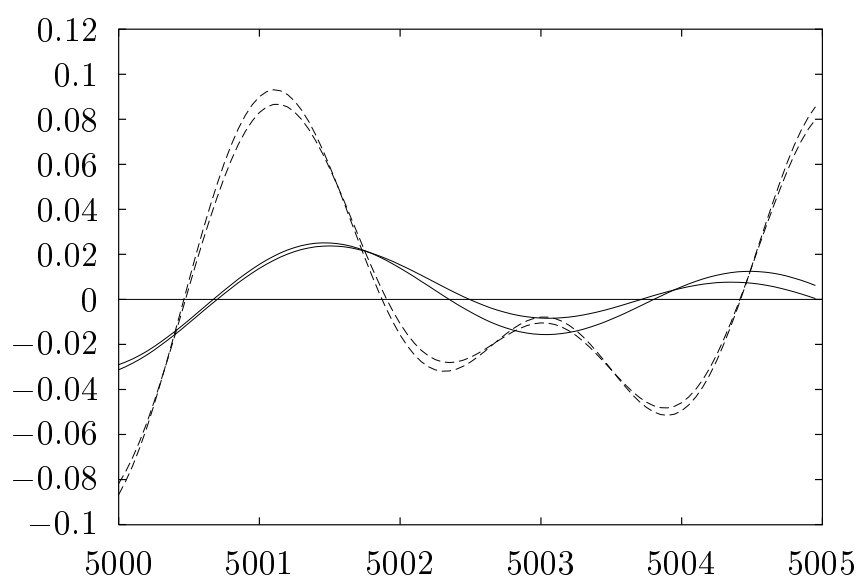

FIGURE 33. Graphs of $(6.2)$ as a function of $x$, showing the effect of a change in $N$. On the left we used $N=10,000$ and 20,000; on the right, 50,000 and 100,000. The broken line is $\hat{\mu}_{6}$, the solid line is $\hat{\mu}_{2.1}$. 


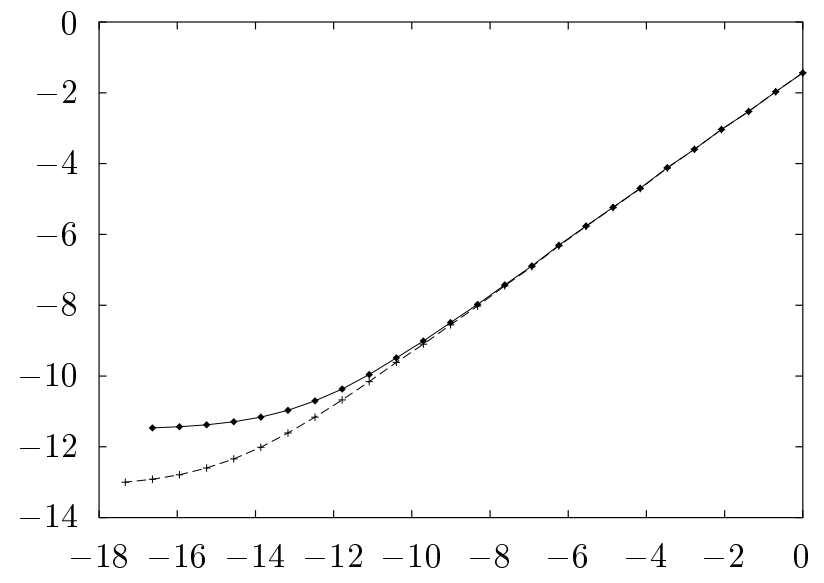

FIGURE 34. Plots of $\log \mathrm{MQV}(s)$ as functions of $\log s$ (logarithms to base 2 ) for $F=\hat{\mu}_{2.1}$, with $\mu_{2.1}$ generated by 100,000 points (upper graph) and 500,000 points (lower graph); compare with Figure 30 . The deviation from a straight line becomes more pronounced for small $s$, but is less severe if we use the more accurate approximation for $\mu$.

\section{REFERENCES}

[Besicovitch 1954] A. S. Besicovitch, Almost Periodic Functions, Dover, New York, 1954.

[Daubechies 1988] I. Daubechies, "Orthonormal bases of compactly supported wavelets", Comm. Pure Appl. Math. 41 (1988), 909-996.

[Daubechies 1992] I. Daubechies, Ten Lectures on Wavelets, CBMS-NSF Regional Conference Series in Applied Mathematics 61, SIAM, Philadelphia, 1992.

[Erdős 1940] P. Erdős, "On the smoothness properties of a family of Bernoulli convolutions", Amer. J. Math. 62 (1940), 180-186.
[Furstenberg and Kesten 1960] H. Furstenberg and H. Kesten, "Products of random matrices", Ann. Math. Statist. 31 (1960), 457-469.

[Hutchinson 1981] J. Hutchinson, "Fractals and selfsimilarity", Indiana Univ. Math. J. 30 (1981), 713747.

[Koksma 1935] J. F. Koksma, "Ein mengentheoretischer Satz über die Gleichverteilung modulo Eins", Compositio Math. 2 (1935), 250-258.

[Lau] K.-S. Lau, "Fractal measures and mean $p$ variations", J. Funct. Anal. 108 (1992), 427-457.

[Lau and Wang] K.-S. Lau and J. Wang, "Mean quadratic variations and Fourier asymptotics of selfsimilar measures", to appear in Monatsh. Math.

[Salem 1983] R. Salem, Algebraic Numbers and Fourier Analysis, Wadsworth, Belmont, CA, 1983.

[Strichartz 1990] R. S. Strichartz, "Self-similar measures and their Fourier transforms I", Indiana Univ. Math. J. 39 (1990), 797-817.

[Strichartz 1992a] R. S. Strichartz, "Self-similarity on nilpotent Lie groups", Contemp. Math. 140 (1992), 123-157.

[Strichartz 1992b] R. S. Strichartz, "Self-similar measures and their Fourier transforms III" (preprint), Cornell University, 1992.

[Strichartz 1993] R. S. Strichartz, "Self-similar measures and their Fourier transforms II", to appear in Trans. Amer. Math. Soc. (1993).

[Wiener and Wintner 1939] N. Wiener and A. Wintner, "On singular distributions", J. Math. Phys. 17 (1939), 233-246. Reprinted as pp. 687-700 in N. Wiener, Collected Works and Commentaries, vol. 2, MIT Press, Cambridge, MA, 1979.

Prem Janardhan, Susquehanna University, Selingrove, PA 17870 (prem@geom.umn.edu)

David Rosenblum, Amherst College, P. O. Box 1647, Amherst, MA 01002 (rosenblu@mtgp2.gs.com)

Robert S. Strichartz, Mathematics Department, White Hall, Cornell University, Ithaca, NY 14853 (math@math.cornell.edu)

Received November 12, 1991; accepted in revised form December 2, 1992 